This item was submitted to Loughborough's Research Repository by the author.

Items in Figshare are protected by copyright, with all rights reserved, unless otherwise indicated.

\title{
Sequential permutation table method for optimization of stacking sequence in composite laminates
}

PLEASE CITE THE PUBLISHED VERSION

http://dx.doi.org/10.1016/j.compstruct.2016.01.052

PUBLISHER

(C) Elsevier

VERSION

AM (Accepted Manuscript)

\section{PUBLISHER STATEMENT}

This work is made available according to the conditions of the Creative Commons Attribution-NonCommercialNoDerivatives 4.0 International (CC BY-NC-ND 4.0) licence. Full details of this licence are available at: https://creativecommons.org/licenses/by-nc-nd/4.0/

\section{LICENCE}

CC BY-NC-ND 4.0

\section{REPOSITORY RECORD}

Jing, Zhao, Qin Sun, and Vadim Silberschmidt. 2019. "Sequential Permutation Table Method for Optimization of Stacking Sequence in Composite Laminates". figshare. https://hdl.handle.net/2134/20489. 


\title{
Sequential permutation table method for optimization of stacking sequence in
} composite laminates

\author{
Zhao Jing $^{1,2}$, Qin Sun ${ }^{1 *}$, Vadim V. Silberschmidt ${ }^{2}$ \\ ${ }^{1}$ School of Aeronautics, Northwestern Polytechnical University, Xi'an 710072, China \\ ${ }^{2}$ Wolfson School of Mechanical and Manufacturing Engineering, Loughborough \\ University, LE11 3TU Leicestershire, UK
}

Abstract: This paper presents a new stacking-sequence optimization method for composite laminates using a multicriteria objective function with buckling, strength and continuity constraint subject to in-plane normal compressive loads. The objective function combines a critical buckling load factor function and a critical failure load function into a single function to maximizes in which the relative influence of a stacking sequence and number of ply orientations is reflected. The buckling-load and failure-load factors are sorted from maximum to minimum respectively in a sequential permutation table. A sequence-design strategy and a ply-orientation selection strategy are developed to identify the ply orientation of each stacking position and number of ply orientations of the stacking sequence involving continuity constraints based on the sequential permutation table. A benchmark problem is presented to illustrate the accuracy and effectiveness of the method; the detailed results are compared with various heuristic methods.

Keywords: Sequential permutation table; Stacking sequence optimization; Laminates; Mechanical properties; Buckling; 


\section{Introduction}

Composite materials provide unmatched potential and large freedom in design to reduce their weight and/or to improve their performance. Thickness, a stacking sequence and ply orientations are often set as design variables to tailor global levels of stiffness and strength of composite laminates $[1,2]$. A discrete set of ply laminates makes it a non-convex, nonlinear and high-dimensional complex mixed-discrete optimization problem [3] with many local optima, thus bringing new challenges for the design and optimization process.

Though the stacking-sequence optimization is a mixed-discrete and high-dimensional optimization problem with discrete variables, modern mathematical optimization methods combined with specific techniques appears to be capable of providing efficient solution to this problem. Buckling is one of the most important constraints in composite structural optimization since it is one of the most common failure forms of composite structures $[2,4]$. A variety of optimization methods were applied to this problem $[5,6]$. The most popular method, namely a genetic algorithm (GA) [7-9], is used in stacking-sequence design with buckling, strength and continuity constraints. The problem, described in [7], of optimization of the buckling load factor by changing the ply orientations, attracts many researchers developing a variety of heuristic methods [8-20]. In particular, a permutation GA with a repair strategy was developed [12] for continuity constraints. Furthermore, a bi-level optimization scheme based on the permutation GA is proposed for the composite wing box design $[14,15]$. An integrated approach, combining a shape optimization process with the 
GA was proposed in [21] for shape optimization and stacking-sequence design of composite laminates. Another popular method is evolutionary algorithm (EA), used in multiobjective optimization of composite laminates [22] and tapered composite structures [23]. More recently, a permutation search (PS) is proposed in [24], where a buckling load factor was expressed as discrete forms of function of ply orientations to reduce computational cost.

On the other hand, optimization of multi-panel composite structures is more difficult due to blending of adjacent panels and nature of ply drops in design. The GA coupled with a response surface method was used in [18] to optimize both single-panel and two-patch design examples. In recent years, a stacking-sequence table (SST) scheme was specialized for EA-based blending optimization [23]. Recently, a global shared-layer blending (GSLB) [25] method was proposed based on a shared-layer blending (SLB) method [26] for blending design.

Although, various heuristic methods are applied to the stacking-sequence optimization problem, they are sensitive to initial values and predefined parameters. For specific optimization problems, the predefined parameters should be adjusted properly to find the optimal solutions. Unsuitable initial values or predefined parameters may result in a low convergence rate or even fail to find the optimum. Additionally, the design space increases exponentially with the increasing number of design variables, making heuristic methods computationally expensive for solving optimization problems of large-scale composite structures. In order to overcome these limitations as well as concerns of convergence difficulties and of problem size of 
heuristic methods, a specific method is motivated by previous study for stacking-sequence optimization.

It is suggested that a stacking sequence of the laminate can be designed based on the classical laminate theory employing a specific designed algorithm [24, 27, 28]. In [27], a two-level method was developed to determine the feasible region of the lamination parameters. Recently, a bi-level optimization scheme for finding an optimal stacking sequence of composite laminates subjected to mechanical, blending and manufacturing constraints was developed in [28]. In this paper, based on a theory of lay-up optimization $[27,28]$, a sequential permutation table (SPT) method was proposed for stacking-sequence optimization.

The remainder of this paper is arranged as follows: in Section 2, an optimization problem is formulated. Then, the SPT method is developed in Section 3. In Section 4, performance of the method is compared with several heuristic methods via a benchmark problem. Finally, some conclusions and suggestions are provided in

\section{Section 5.}

\section{Statement of optimization model}

Buckling analysis of a symmetric and balanced composite plate simply supported on four edges (Fig. 1a), can be formulated using an analytical method [16]. The classical plate theory of composite laminate is briefly summarized in Appendix A. Considering an orthotropic plate, where $D_{16}=D_{26}=0$, buckling of the plate into $m$ and $n$ half-waves (along the $x$ and $y$ directions) occurs when the load amplitude factor $\lambda_{b}$ reaches the following value [16]: 


$$
\lambda_{c b}=\pi^{2} \frac{\left[D_{11}\left(\frac{m}{a}\right)^{4}+2\left(D_{12}+2 D_{66}\right)\left(\frac{m}{a}\right)^{2}\left(\frac{n}{b}\right)^{2}+D_{22}\left(\frac{n}{b}\right)^{4}\right]}{\left[F_{x}\left(\frac{m}{a}\right)^{2}+F_{y}\left(\frac{n}{b}\right)^{2}\right]}
$$

where $F_{\mathrm{x}}$ and $F_{\mathrm{y}}$ are the normal compressive loads applied to the plate in $x$ and $y$ directions, respectively. Substituting Eq. (A.1) into Eq. (1)

$$
\lambda_{c b}=\sum_{k=1}^{N / 2}\left(\lambda_{c b}\right)_{k}=\frac{\pi^{2}}{F_{x}\left(\frac{m}{a}\right)^{2}+F_{y}\left(\frac{n}{b}\right)^{2}} \sum_{k=1}^{N / 2}\left[\left(D_{11}\right)_{k}\left(\frac{m}{a}\right)^{4}+2\left(\left(D_{12}\right)_{k}+2\left(D_{66}\right)_{k}\right)\left(\frac{m}{a}\right)^{2}\left(\frac{n}{b}\right)^{2}+\left(D_{22}\right)_{k}\left(\frac{n}{b}\right)^{4}\right](2)
$$

In Eq. (2), $\lambda_{c b}$ is expressed as the sum of $\left(\lambda_{c b}\right)_{k}$, which is a linear function of the flexural stiffness parameters $\left(D_{\mathrm{ij}}\right)_{k}$. Obviously, for a specified stacking position $k$, the ply orientation $\theta_{\mathrm{k}}$ is the only design variable in the optimization of flexural stiffness parameters and, thus, superposition principles are suitable for evaluation of the buckling load factor $\lambda_{c b}$ [24]. As a result, the maximizing of $\lambda_{c b}$ is equivalent to identification of the ply orientation $\theta_{\mathrm{k}}$ at stacking position $k$. To simplify the optimization and design processes, the buckling load factor is formulated as a linear function of the stacking sequence $[24,28]$, therefore, the stacking sequence can be designed linearly, significantly reducing the computational cost.

Based on the aforementioned analysis, several important features of stacking-sequence design for the maximum buckling load can be summarized:

(I) At each stacking position $k$, the optimal orientation $\theta_{\mathrm{k}}$ can be identified separately. (II) For each part of the laminates, the stacking sequence can be formulated as independent variable. In other words, the stacking sequence can be designed block by block.

(III) Compared to the plies near the mid-plane, the outermost plies make a major 
contribution to the buckling performance.

The above features imply that the sequence of the laminate can be designed linearly from the mid-plane to the outermost layer by selecting the proper ply orientation for maximizing the buckling load factor.

On the other hand, the failure load of the plate can be evaluated with the first-ply failure approach based on the maximum strain criterion [16]. Principal strains in the $k$ th layer of the plate are related to the loads by the following relations

$$
\begin{aligned}
& \lambda F_{x}=A_{11} \varepsilon_{x}+A_{12} \varepsilon_{y}, \\
& \lambda F_{y}=A_{12} \varepsilon_{x}+A_{22} \varepsilon_{y}, \\
& \lambda F_{x y}=A_{66} \gamma_{x y},
\end{aligned}
$$

where $\lambda$ is the load factor, $\varepsilon_{\mathrm{x}}, \varepsilon_{\mathrm{y}}$ and $\gamma_{\mathrm{xy}}$ are the global strain components. The strains for each orientation are given as

$$
\begin{aligned}
& \varepsilon_{1}^{k}=\cos ^{2} \theta_{k} \varepsilon_{x}+\sin ^{2} \theta_{k} \varepsilon_{y}+\cos \theta_{k} \sin \theta_{k} \gamma_{x y}, \\
& \varepsilon_{2}^{k}=\sin ^{2} \theta_{k} \varepsilon_{x}+\cos ^{2} \theta_{k} \varepsilon_{y}-\cos \theta_{k} \sin \theta_{k} \gamma_{x y}, \\
& \gamma_{12}^{k}=\sin 2 \theta_{k}\left(\varepsilon_{y}-\varepsilon_{x}\right)+\left(\cos ^{2} \theta_{k}-\sin ^{2} \theta_{k}\right) \gamma_{x y},
\end{aligned}
$$

where $\varepsilon_{1}, \varepsilon_{2}$, and $\gamma_{12}$ are local strains of each orientation.

The strength failure load factor $\lambda_{c f}$ is taken to be the largest load factor $\lambda$ given by Eq. (3), which corresponds to the critical value when one of the local strains $\varepsilon_{1}, \varepsilon_{2}$, and $\gamma_{12}$ of all layers reaches the ultimate allowable strain value: $\varepsilon_{1}^{u a}=0.008, \varepsilon_{2}^{u a}=0.029$ and $\gamma_{12}^{\mu a}=0.015$ with a given safety factor 1.5 [29]. Therefore, the failure load factor $\lambda_{c f}$ can be evaluated as

$$
\lambda_{c f}=\frac{1}{q} \min \left\{\frac{\varepsilon_{1}^{u a}}{\varepsilon_{1 \max }}, \frac{\varepsilon_{2}^{u a}}{\varepsilon_{2 \max }}, \frac{\gamma_{12}^{u a}}{\gamma_{12 \max }}\right\}
$$

$\varepsilon_{1 \max }, \varepsilon_{2 \max }$ and $\gamma_{12 \max }$ are the maximum strains and the safety factor $q=1.5$.

To prevent failure, the objective function should be taken to the maximum of the 
smaller critical load factor $\lambda_{c}=\min \left(\lambda_{c b}, \lambda_{c f}\right) \quad[16]$ :

$$
\lambda_{c}=\max \left(\min \left(\lambda_{c b}, \lambda_{c f}\right)\right)
$$

The objective of this work is to develop a method to maximize the critical load factor $\lambda_{c}$ by changing the ply orientations. The continuity constraints are also applied to the optimization, limiting the continuous number of plies of the same orientations to 4 to avoid matrix cracking.

\section{Sequential permutation table method}

\subsection{Design criterion}

It is observed that feasible regions of lamination parameters are convex and their values reach a vertex with one ply orientation at all stacking positions [27, 28]. Such rules can be applied to flexural stiffness thanks to their linear dependence on lamination parameters. Furthermore, the buckling load factor $\lambda_{c b}$ is a linear function of flexural stiffness parameters and reaches an extreme value with one ply orientation at all stacking positions. Therefore, $\lambda_{c b}$ can be designed to vary linearly by changing the orientation ply by ply. The expression $\lambda_{c b}(\theta)$ denotes the buckling factor $\lambda_{c b}$ (Eq.(2)), with all stacking positions setting as $\theta$.

Since $\lambda_{c b}$ is a convex-hull function of the ply-orientation variable $\theta$ (Fig. 2a), the optimal stacking sequence for the maximal buckling load factor $\lambda_{c b}$ can be easily obtained [28]. On the contrary, the optimum number of the ply orientations for the maximal failure load factor $\lambda_{c f}$ is a non-convex hull function of the number of ply orientations (Fig. 2b). To obtain the optimal sequence of the laminate with buckling and strength constraints, these two load factors are coupled via the objective function 
(Eq. (6)), thus, the optimal stacking-sequence can be designed under the control of linear regularity of $\lambda_{c b}$ (Fig.2c).

Accordingly, a sequential permutation table (SPT) method is proposed as follows. Assume that there are $M$ orientations $\left\{\theta_{1}, \theta_{2}, \ldots, \theta_{\mathbf{M}}\right\}$ in a symmetric and balanced laminate with a total of $N$ plies. First, set the ply orientation to be the same $\theta$ at all stacking positions if $\theta=0^{\circ}$ or $90^{\circ}$; Otherwise, $\theta$ and $-\theta$ should be a half (due to balance requirement) in the laminate and a pair of $\pm \theta$ should stack together (Fig. 3). As shown in case 2 of Fig. 3, the buckling load factor and failure load factor of the stacking sequence are equal to that of setting all of the orientations to be $\theta$ because these factors are even functions of ply orientation. Thus, there are $[M / 2]+1$ cases of different load factors by setting different ply orientations $\theta \in\left\{\theta_{1}, \theta_{2}, \ldots, \theta_{\mathrm{M}}\right\}$ to be the same in the laminate. The values of $\lambda_{c b}$ and $\lambda_{c f}$ can be evaluated for all cases according to Eq. (2) and Eq. (5) respectively, and sorted in two columns in Table 1. The first column is sorted by the value of $\lambda_{c b}$ from maximum to minimum, where $\lambda_{c b}\left(\alpha_{\max }\right)>\ldots>$ $\lambda_{c b}(\alpha)>\ldots>\lambda_{c b}\left(\alpha_{\min }\right)$; The second column is sorted by the value of $\lambda_{c f}$ from maximum to minimum, where $\lambda_{c f}\left(\beta_{\max }\right)>\ldots>\lambda_{c f}(\beta)>\ldots>\lambda_{c f}\left(\beta_{\min }\right)$. Note that only a half of the number of plies $n=N / 2$ is used to characterize the symmetric and balanced laminate with $N$ plies.

Additionally, if ply orientations are not given or produced at random, the best orientation for maximizing $\lambda_{c b}$ should be identified first. Since $\lambda_{c b}$ is a convex function of ply orientations of stacking positions, a numerical optimization method (e.g. a bisection method) can be applied to find the best orientation for maximizing $\lambda_{c b}$. If the 
best orientation $\theta_{\max }$ is found, candidate orientations should be set as $\left\{\theta_{\max }-90\right.$, $\left.\theta_{\max }-45, \theta_{\max }, \theta_{\max }+45\right\}$. The candidate orientations are decided by the number of the orientations set in design, set here as $M$ and $M=4$. Thus, the SPT can be obtained by evaluation of $\lambda_{c b}$ and $\lambda_{c f}$ for each candidate orientation. In engineering, the candidate orientations are often set as $\left\{0^{\circ}, 45^{\circ},-45^{\circ}, 90^{\circ}\right\}$ or $\left\{0^{\circ}, \pm 15^{\circ}, \pm 30^{\circ}, \pm 45^{\circ}, \pm 60^{\circ}, \pm 75^{\circ}\right.$, $90^{\circ}$ \}, so the SPT can be easily obtained.

The sequential permutation table is a guide for the sequence-optimization process, it will be used in the sequence-design and ply-orientation selection strategies.

\subsection{Sequence-design strategy}

In case of all constraints of buckling, strength and continuity involved simultaneously, $\left(\lambda_{c b}\right)_{\max }$ can decrease because of variations of the number of the ply orientations and the stacking sequence. Consequently, strategies to optimize the stacking sequence and select ply orientations should be developed. First of all, assume that the ply orientations are given as $\left\{\theta_{1}, \theta_{2}, \cdots, \theta_{\mathrm{M}}\right\}$ and the corresponding ply numbers are $\left(N_{\theta 1}, N_{\theta 2}, \cdots, N_{\theta \mathrm{M}}\right)$ in one half of the laminate, respectively. Thus, the stacking position number $n=\sum_{i=1}^{M} N_{\theta i}=N / 2$, then, the optimal sequence providing the maximum buckling load factor can be obtained [28]:

$$
[\underbrace{\theta_{\max } / \ldots / \theta_{\max }}_{N_{\theta_{\max }}} / \ldots / \underbrace{\theta_{i} / \ldots / \theta_{i}}_{N_{\theta_{i}}} / \ldots / \underbrace{\theta_{\min } / \ldots / \theta_{\min }}_{N_{\theta_{\min }}}]_{s}
$$

where, the plies with the same orientation are adjacent to the ply with number $N_{\theta \mathrm{i}}(1 \leq$ $i \leq M)$ and $\lambda_{c b}\left(\theta_{\max }\right)>\ldots>\lambda_{c b}\left(\theta_{\mathrm{i}}\right)>\ldots>\lambda_{c b}\left(\theta_{\min }\right)$. However, the continuity constraint is always violated in Eq. (7), thus, an appropriate adjustment should be made in the 
sequence. The repair strategy given in [24] is applied here to deal with continuity constraints. The sequence-design strategy with given numbers of each orientation is described in detail as following and an example is shown in Fig. 4.

Step 1: Initialize the sequence according to Eq. (7) based on the numbers of ply orientations $\left(N_{\theta 1}, N_{\theta 2}, \cdots, N_{\theta \mathrm{M}}\right)$. Evaluate the initial values of $\lambda_{c b}, \lambda_{c f}$ and $\lambda_{c}$.

Step 2: Detect the violated positions moving from the mid-plane to the outermost ply

(Fig. 4), and save the results in an array Vol_pos. If the numbers of violated positions are different, the one with fewer violated positions (Vol_pos1 in Fig. 4) should be chosen. On the contrary, if the numbers of violated positions are the same (Vol_pos2 and Vol_pos3 in Fig. 4), the one close to the mid-plane (Vol_pos3) should be chosen.

Step 3: Exchange the orientations of violated positions with different orientations and those close to mid-plane one by one from the outermost ply to the mid-plane until the sequence satisfies the continuity constraints. Evaluate $\lambda_{c b}, \lambda_{c f}$ and $\lambda_{c}$. Particularly, repeat Step 2 after Step 3 since the violated positions may change after the exchange operation.

In Fig. 4, the final optimal sequence is Seq9. Algorithm 1 gives the detailed computer program of the sequence design strategy, where angle denotes an orientation at a position of the sequence.

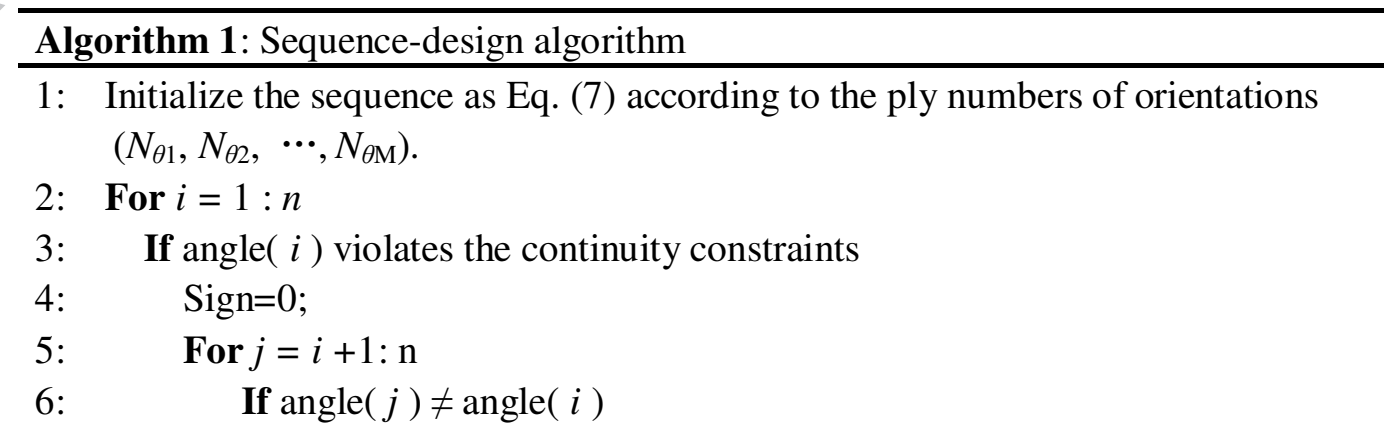




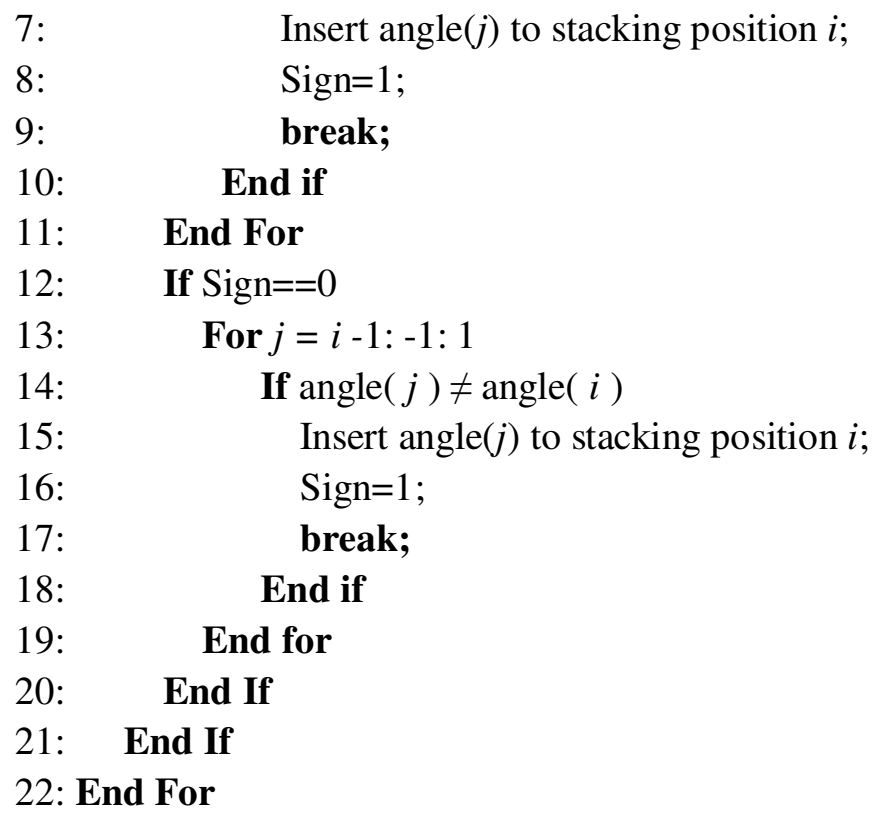

\subsection{Ply-orientation selection strategy}

In order to maximize the objective function $\lambda_{c}, \lambda_{c b}$ and $\lambda_{c f}$ should be maximized simultaneously. However, Eq. (7) only gives the maximum $\lambda_{c b}$. To improve $\lambda_{c f}$, the number of ply orientations and stacking sequence of laminate should be adjusted simultaneously. Based on the analysis of Section 2, three criteria should be satisfied during adjustment, where $\alpha_{\max }$ and $\beta_{\max }$ are the orientations in SPT (Table 1).

(1). Since the initialization of the sequence has maximal $\lambda_{c b}$, the adjustments of the sequence should be as few as possible to keep $\lambda_{c b}$ while improve $\lambda_{c f}$. Thus, the adjustments should start from the mid-plane to the outermost position.

(2). To improve $\lambda_{c f}$, the orientation $\beta_{\max }$ with maximal $\lambda_{c f}$ should replace $\alpha_{\max }$ sequentially from the mid-plane to the outermost ply until $\lambda_{c f}$ reaches and exceeds $\lambda_{c b}$

(3). To further improve $\lambda_{c}$, other orientations should be used to replace $\alpha_{\max }$ and $\beta_{\max }$ according to the sequential permutation table at proper stacking positions. In 
addition, the balance and continuity constraints should be considered in each replacement.

On the basis of these three points, a ply-orientation selection strategy used to maximize the objective $\lambda_{c}$ is proposed based on the sequential permutation table, where the levels of thickness and ply orientations are identified while the number of ply orientations and stacking sequence are set as design variables. The detailed scheme of the ply-orientation selection strategy is described as follows and the flowchart is demonstrated in Fig. 5.

Step 1: Initialize the sequential permutation table (SPT) by the predefined ply orientations $\left(\theta_{1}, \theta_{2}, \ldots, \theta_{\mathrm{M}}\right)$. Set the orientation $\alpha_{\max }$ at all stacking positions. Save the sequence as "Seq" together with its corresponding $\lambda_{c b}, \lambda_{c f}$ and $\lambda_{c}$. According to the $\mathrm{SPT}, \lambda_{c b}>\lambda_{c f}$ in the initial sequence "Seq".

Step 2: Operation: Using $\beta_{\max }$ to replace $\alpha_{\max }$ from the mid-plane to the outermost ply, set the current position as $k$ and the current sequence as "Seq". At each position $k$, the following two judgments should be considered.

Judgment a: if $\alpha_{\max } \in\left\{0^{\circ}, 90^{\circ}\right\}$ and $\beta_{\max } \in\left\{0^{\circ}, 90^{\circ}\right\}$, only one position needs to be replaced each time, then $k=k-1$; otherwise, two connecting positions need to be replaced to satisfy the balance constraints, then $k=k-2$.

Judgment b: if the current position violates the continuity constraints, jump to the next position $k=k-1$;

After each replacement, evaluate the $\lambda_{c b}, \lambda_{c f}$ and $\lambda_{c}$ of the current sequence "Seq" and save the sequence with its corresponding factors. 
Step 3: Judgment: for the current sequence "Seq" check: $\lambda_{c f}>\lambda_{c b}$ ? If it is, turn to Step 4; otherwise, $k=k-1$, turn to Step 2.

Step 4: Set the last sequence that satisfies $\lambda_{c f}<\lambda_{c b}$ as "Seq1" with its corresponding $\lambda_{c b 1}, \lambda_{c f 1}, \lambda_{c 1}$ and $\left(\lambda_{c 1}\right)_{\text {old }}=\lambda_{c 1}$, then turn to Step 5; Set the current sequence that satisfies $\lambda_{c f}>\lambda_{c b}$ as "Seq2" with its corresponding $\lambda_{c b 2}, \lambda_{c f 2}, \lambda_{c 2}$ and $\left(\lambda_{c 2}\right)_{\text {old }}=\lambda_{c 2}$, then turn to Step 7.

Step 5: In "Seq1": $\lambda_{c f 1}<\lambda_{c b 1}, \lambda_{c f 1}$ can be improved by selecting an orientation $\beta_{1}$ from the SPT, which satisfies the condition $\lambda_{c f 1}\left(\beta_{\max }\right)>\lambda_{c f 1}\left(\beta_{1}\right)>\lambda_{c f 1}\left(\alpha_{\max }\right)$. If several orientations satisfy $\lambda_{c f 1}\left(\beta_{\max }\right)>\lambda_{c f 1}\left(\beta_{1}\right)>\lambda_{c f 1}\left(\alpha_{\max }\right), \beta_{1}$ should be the one that maximizes $\lambda_{c 1}$. Use $\beta_{1}$ to replace $\alpha_{\max }$ at position $k$. Save it as "Seq1" with its corresponding factors $\lambda_{c b 1}, \quad \lambda_{c f 1}$ and $\lambda_{c 1}$. Record the new $\lambda_{c 1}$ as $\left(\lambda_{c 1}\right)_{\text {new }}$.

Step 6: Compare $\left(\lambda_{c 1}\right)_{\text {new }}$ with $\left(\lambda_{c 1}\right)_{\text {old. }}$ If $\left(\lambda_{c 1}\right)_{\text {new }}<\left(\lambda_{c 1}\right)_{\text {old }}$, the replaced operation failed, and $\left(\lambda_{c 1}\right)_{\text {old }}$ is the optimal solution. Save this optimal sequence as "Opt_seq1" and its corresponding factors $\lambda_{c b 1}, \lambda_{c f 1}$ and $\lambda_{c 1}$, turn to Step 11; otherwise, the replacement operation succeeded, and the objective $\lambda_{c 1}$ can be improved further. Compare $\lambda_{c b 1}$ with $\lambda_{c f 1}$. If $\lambda_{c b 1}>\lambda_{c f 1}, k=k-1$, turn to Step 5; otherwise, $\lambda_{c b 1}<\lambda_{c f 1}, k=k-1$, turn to Step 7 .

Step 7: In "Seq2": $\lambda_{c f 2}>\lambda_{c b 2}, \lambda_{c b 2}$ can be further improved by selecting the orientation $\alpha_{1}$ from SPT which satisfies $\lambda_{c b 2}\left(\alpha_{\max }\right)>\lambda_{c b 2}\left(\alpha_{1}\right)>\lambda_{c b 2}\left(\beta_{\max }\right)$. Use the orientation $\alpha_{1}$ to replace $\beta_{\max }$ without identifying the sequence. Calculate the new $\lambda_{c f 2}$. Record the new $\lambda_{c f 2}$ as $\left(\lambda_{c f 2}\right)_{\text {new }}$

Step 8: If $\left(\lambda_{c f 2}\right)_{\text {new }}<\left(\lambda_{c 2}\right)_{\text {old }}$, the replacement operation failed. Delete the candidate orientation, turn to Step 10; otherwise, $\left(\lambda_{c f 2}\right)_{\text {new }}>\left(\lambda_{c 2}\right)_{\text {old }}$, save the number of the ply 
orientations, turn to Step 9.

Step 9: Using the sequence-design strategy to optimize the sequence, where the number of ply orientations was identified. Save all the optimized sequences. Select the sequence with the maximal $\lambda_{c}$, save the sequence as "Seq2" with corresponding $\lambda_{c b 2}, \lambda_{c f 2}$ and $\lambda_{c 2}$. Record the new $\lambda_{c 2}$ as $\left(\lambda_{c 2}\right)_{\text {new. }}$

Step 10: Compare $\left(\lambda_{c 2}\right)_{\text {new }}$ with $\left(\lambda_{c 2}\right)_{\text {old. }}$ If $\left(\lambda_{c 2}\right)_{\text {new }}<\left(\lambda_{c 2}\right)_{\text {old }}$, the replacement operation failed, and $\left(\lambda_{c 2}\right)_{\text {old }}$ is the optimal solution. Save this optimal sequence as "Opt_seq2" and its corresponding factors $\lambda_{c b 2}, \lambda_{c f 2}$ and $\lambda_{c 2}$, turn to Step 11; otherwise, the replacement operation succeeded. Compare $\lambda_{c b 2}$ with $\lambda_{c f 2}$. If $\lambda_{c b 2}<\lambda_{c f 2}, k=k-1$, turn to Step 7; otherwise, $\lambda_{c b 2}>\lambda_{c f 2}, k=k-1$, turn to Step 5 .

Step 11: Two optimal solutions "Opt_seq1" and "Opt_seq2" are obtained. The sequence-design strategy is applied to these two sequences to overcome the continuity constraints. Compare the values of $\lambda_{c}$, if $\left(\lambda_{c}\right)_{\text {Opt_seq1 }}>\left(\lambda_{c}\right)_{\text {Opt_seq2 }}$, the optimal sequence is "Opt_seq1"; otherwise, $\left(\lambda_{c}\right)_{\text {Opt_seq1 }}<\left(\lambda_{c}\right)_{\text {Opt_seq2 }}$, the optimal sequence is "Opt_seq2"

Algorithm 2 gives the detailed computer program of the ply-orientation selection strategy, where angle denotes an orientation at a position of the sequence.

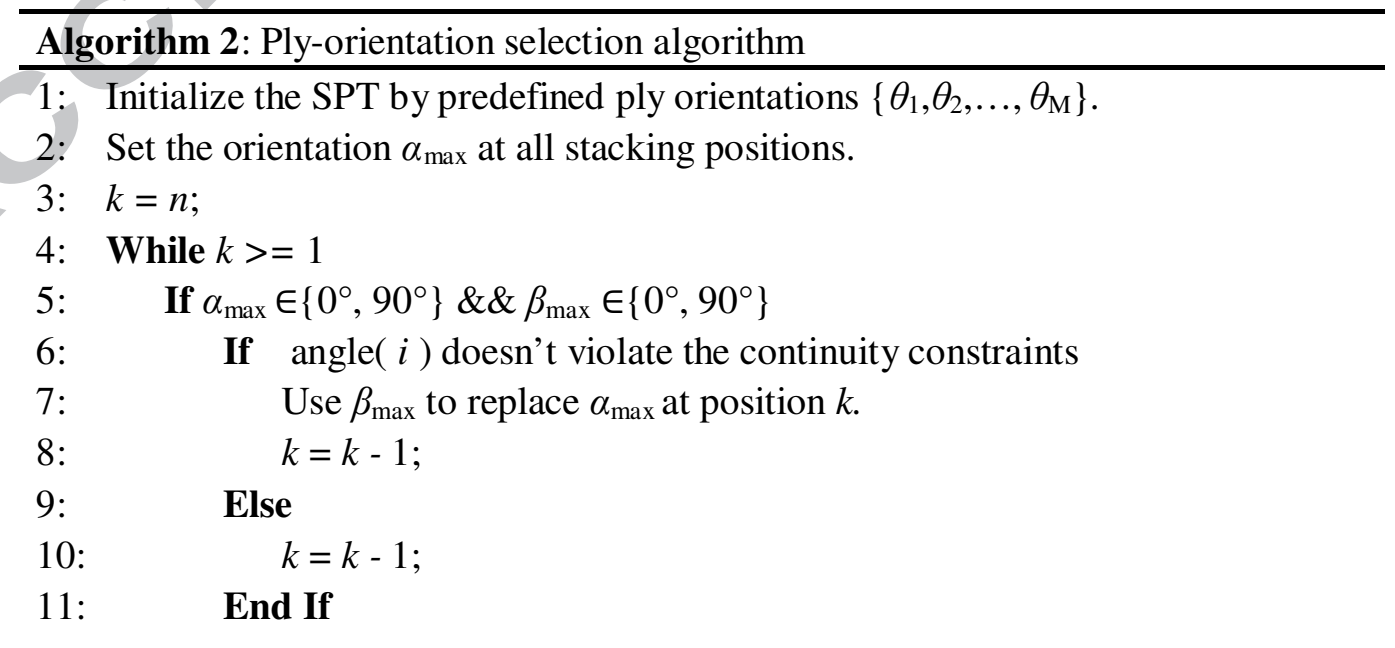


12: $\quad$ Else

13: If angle( $i$ ) doesn't violate the continuity constraints

14: $\quad$ Use a pair of $\beta_{\max }$ to replace a pair of $\alpha_{\max }$ at position $k$ and $k-1$.

15: $\quad k=k-2$;

16: $\quad$ Else

17: $\quad k=k-1$;

18: $\quad$ End If

19: $\quad$ End If

20: Evaluate $\lambda_{c b}, \lambda_{c f}$ and $\lambda_{c}$ of the current sequence "Seq".

21: If $\lambda_{c f}>=\lambda_{c b}$

22: Set the last sequence which satisfies $\lambda_{c f}<\lambda_{c b}$ as "Seq1" and get its $\lambda_{\mathrm{cb} 1}$, $\lambda_{\mathrm{cf} 1}, \lambda_{\mathrm{c} 1}$ and $\left(\lambda_{c 1}\right)_{\mathrm{old}}=\lambda_{\mathrm{c} 1}$

23: $\quad$ Select an orientation $\beta_{1}$ in SPT: $\lambda_{c f 1}\left(\beta_{\max }\right)>\lambda_{c f 1}\left(\beta_{1}\right)>\lambda_{c f 1}\left(\alpha_{\max }\right)$.

24: $\quad \beta_{1}=\max \left\{\beta_{1} \in\left\{\lambda_{c f 1}\left(\beta_{\max }\right)>\lambda_{c f 1}\left(\beta_{1}\right)>\lambda_{c f 1}\left(\alpha_{\max }\right)\right\}\right\}$.

25: $\quad$ Use $\beta_{1}$ to replace $\alpha_{\max }$ at position $k$.

26: $\quad$ Save it as "Seq1" with its corresponding factors $\lambda_{c b 1}, \lambda_{c f 1}, \lambda_{c 1}$ and $\left(\lambda_{c 1}\right)_{\text {new }}=\lambda_{c 1}$.

27: $\quad$ If $\left(\lambda_{c 1}\right)_{\text {new }}<\left(\lambda_{c 1}\right)_{\text {old }}$

28: $\quad$ The replacement operation failed, $\left(\lambda_{c 1}\right)_{\text {old }}$ is the optimal solution.

Save this optimal

29: $\quad$ sequence as "Opt_seq1" and its corresponding factors $\lambda_{c b 1}, \lambda_{c f 1}$ and $\lambda_{c 1}$, turn to row 67 ;

30:

31:

32:

33:

34:

35:

36:

37:

Else

$$
\text { If } \begin{gathered}
\lambda_{c b 1}>\lambda_{c f 1} \\
k=k-1
\end{gathered}
$$

Turn to row 22.

Else

$$
k=k-1 ;
$$

Turn to row 40.

\section{0:}

\section{End If}

\section{End If}

Set the last sequence which satisfies $\lambda_{c f}>\lambda_{c b}$ as "Seq2" and get its $\lambda_{c b 2}$, $\lambda_{c f 2}, \lambda_{c 2}$ and $\left(\lambda_{c 2}\right)_{\text {old }}=\lambda_{c 2}$;

41: $\quad$ Select orientations $\alpha_{1}$ in SPT: $\lambda_{c b 2}\left(\alpha_{\max }\right)>\lambda_{c b 2}\left(\alpha_{1}\right)>\lambda_{c b 2}\left(\beta_{\max }\right)$

42: $\quad$ Use the orientation $\alpha_{1}$ to replace $\beta_{\max }$ without identifying the stacking sequence.

43: Calculate the new $\lambda_{c f 2}$ according to the number of ply orientations and $\left(\lambda_{c f 2}\right)_{\text {new }}=\lambda_{c f 2}$.

44: $\quad$ If $\left(\lambda_{c f 2}\right)_{\text {new }}<\left(\lambda_{c 2}\right)_{\text {old }}$

45: $\quad$ The replacement operation failed and delete the candidate orientation $\alpha_{1}$, turn to row 54 ;

46: $\quad$ Else 
47: $\quad$ Save the number of ply orientations.

48: $\quad$ End If

49: $\quad$ Call Algorithm 1 to optimize the sequence with the obtained number of ply orientations.

50: $\quad$ Save all the optimized sequence.

51: $\quad$ Select the sequence with maximal $\lambda_{c}$, save the sequence as "Seq2" with corresponding $\lambda_{c b 2}, \lambda_{c f 2}$,

52: $\quad \lambda_{c 2}$ and $\left(\lambda_{c 2}\right)_{\text {new }}=\lambda_{c 2}$.

53: $\quad$ If $\left(\lambda_{c 2}\right)_{\text {new }}<\left(\lambda_{c 2}\right)_{\text {old }}$

54: $\quad$ The replacement operation failed and $\left(\lambda_{c 2}\right)_{\text {old }}$ is the optimal solution. Save this optimal

55: $\quad$ sequence as "Opt_seq2" and its corresponding factors $\lambda_{c b 2}, \lambda_{c f 2}$ and $\lambda_{c 2}$, turn to row 67.

56: $\quad$ Else

57: $\quad$ If $\lambda_{c b 2}<\lambda_{c f 2}$,

58: $\quad k=k-1$,

59: $\quad$ turn to row 22;

60: $\quad$ Else

61: $\quad k=k-1$,

62: $\quad$ turn to row $\mathbf{4 0 .}$

63: $\quad$ End If

64: $\quad$ End If

65: $\quad$ End If

66: End While

67: Call Algorithm 1 to optimize the sequence "Opt_seq1" and "Opt_seq2".

68: If $\left(\lambda_{c}\right)_{O p t_{-} \text {seq }}>\left(\lambda_{c}\right)_{\text {Opt_seq2 }}$

69: The optimal sequence is "Opt_seq1";

70: Else

71: The optimal sequence is "Opt_seq2".

72: End If

\section{Numerical experiments}

A benchmark problem proposed by Le Riche and Haftka [7], which was optimized with various methods in $[8-11,13,16,19,20]$, is used here to validate the SPT method. The material properties of a graphite-epoxy plate are given [7] as: $E_{1}=127.59$ $\mathrm{GPa}, E_{2}=13.03 \mathrm{GPa}, G_{12}=6.41 \mathrm{GPa}, t=0.0127 \mathrm{~cm}, v_{12}=0.3$. In this work, four additional load cases with the aspect ratio $a / b=0.125$ and 64 layers are added (Table 
2). Furthermore, to verify the efficiency of this method, the orientations are expanded to 12 . The sets of orientations for 4 and 12 are introduced as $\left(0^{\circ}, 45^{\circ},-45^{\circ}, 90^{\circ}\right)$ and $\left(\left(0^{\circ}, \pm 15^{\circ}, \pm 30^{\circ}, \pm 45^{\circ}, \pm 60^{\circ}, \pm 75^{\circ}, 90^{\circ}\right)\right.$, respectively. At the same time, a comparison between the results of $\lambda_{c}$ for 4 and 12 orientations is made.

The optimization results for 4 and 12 orientations are given in Tables 3 and 4 respectively, and their searching process is shown in Fig. 6. To clarify the searching process of the SPT method, the load case 2 with 4 orientations $\left(0^{\circ}, 45^{\circ},-45^{\circ}, 90^{\circ}\right)$ is chosen as the example to demonstrate the sequential permutation table method in Appendix B. A comparison of the optimal results of 4 and 12 orientation is demonstrated in Fig. 7. The searching process of the SPT method starts from the maximal vertex of the buckling load factor [27] instead of from different initial points. In Tables 3 and 4, the optimal results demonstrate that same orientations prefer to stack together and those not close to them are adjusted to satisfy the continuity constraints. The number of evaluations of the load factors does not exceed 70, and it is noticed that the evaluations of $\lambda_{c b}$ and $\lambda_{c f}$ are different. This is caused by deletion of orientations at Step 8 of the ply-orientation selection strategy since no better solutions can be obtained for these orientations. In Fig. 6, only the results of evaluations of $\lambda_{c f}$ and its corresponding $\lambda_{c b}$ are presented, while those for the factors in the SPT and the number of evaluations for any single factor are not included since the objective value $\lambda_{c}$ requires evaluations of $\lambda_{c f}$ and $\lambda_{c b}$ simultaneously. Furthermore, it is observed that $\lambda_{c b}$ decreases slowly, while $\lambda_{c f}$ increases fast at the initial stages of evaluation because the adjustments start from the mid-plane, having minor influence on $\lambda_{c b}$ while 
improving $\lambda_{c f}$ fast. Then, the values of load factors cross, other orientations are added to improve the objective $\lambda_{c}$ with the ply-orientation selection strategy. By comparing the results in Tables 3 and 4, it is noticed that as the ply orientations increase, the $\lambda_{c}$ increases, as shown in Fig. 7. At the same time, as the orientations increase, the design space is increased exponentially, while the number of evaluations does not increase significantly. This demonstrates the potential of the SPT method in solving optimization problems for large-scale composite structures.

Then, at the next stage of our analysis, the first three load cases, that were also studied by using various methods $[8-11,16,19,20]$, were selected to validate efficiency of the proposed method in terms of computational cost and reliability. The convergence rate of GA is sensitive to the predefined parameters, such as: population size, genetic operators, crossover rate and mutation rate. The crossover rate is often given large in design to produce more different child strings while maintaining their parents' characteristics. On the contrary, the mutation rate is often set small to bring in completely new combinations to protect population against uniformity [9]. Moreover, since the stacking-sequence design problem have many local optimums, the population size is important for search of GA. If the population size is too small, it may prematurely converge to a local optimum; otherwise, if the population size is too big, it is expensive to converge to a global optimum [7]. The accuracy and efficiency of different methods is apparent from results given in Table 5. The reliability is defined as performing 100 optimization runs and checking how many of these runs reached the optimum at any given point. In general, the optimal results of $\lambda_{c}$ as well as 
the efficiency are comparable for most of these heuristic methods. The AGATLA method performs much better than other existing methods in finding the optimal sequence. Also, the AGATLA method can provide superior results since the design space is expanded (without the constraint of two layers stack together). Compared to the AGATLA method, the proposed SPT method not only has a higher convergence speed (about 3 times that of the AGATLA method and about 10 times those of other methods) but also has the highest reliability. The SPT method generally has $100 \%$ reliability, which is superior to all of other methods. Since the SPT method is executed step by step, and the optimum is obtained by comparison of two factors $-\lambda_{c b}$ and $\lambda_{c f}$, it is stopped automatically. For the three load cases here, compared with the previous best results, the objective value $\lambda_{c}$ was improved by $12.39 \%, 0.45 \%$ and $2.94 \%$, respectively.

To decrease the design variable space, in [8-11, 16, 19], it was assumed that two layers were stacked together. In contrast, however, the AGATLA [20] and the SPT methods can release this assumption, which, as a result, increased the design space. Table 6 gives the data for the design space with the corresponding stacking positions and numbers of evaluations in the SPT method. As the stacking position increase from 48 to 64 and orientations increase from 4 to 12 , the design space increases from $4^{24}$ to $12^{32}$, while the number of evaluations in SPT method increases from some 10 to 70. The remarkable reduction of the evaluations is due to the fact that in SPT method the load factor is maximized by designing the stacking sequence linearly from the mid-plane to outermost position. 


\section{Conclusions}

In this work, a sequential permutation table (SPT) method is proposed to optimize the stacking sequence of the composite laminate with buckling, strength and continuity constraints. The ply orientations are set to be the same with the predefined values at all stacking positions. The corresponding sequences are saved in a table, sorted according to the buckling load and failure load factors respectively. Corresponding sequence-design and ply-orientation selection strategies are also developed to design the stacking sequence, which is obtained by adjusting orientations from the mid-plane to the outermost position sequentially, based on the information of the sequential permutation table.

A benchmark problem was chosen as a test case to validate the performance of the proposed method. It is demonstrated that the SPT method not only can considerably reduce the number of evaluations but also can remarkably increase the reliability of the results. The developed method has a great potential in solving high-dimensional discrete-variables stacking-sequence optimization problems for composites structures.

Acknowledgement. This work is supported by the National Natural Science Foundation of China (51375386).

\section{Appendix A Classical plate analysis}

Mechanics of laminates (see an example) in Fig. 1a can be described with the 
classical plate theory [1],

$$
\left\{\begin{array}{c}
F \\
M
\end{array}\right\}=\left[\begin{array}{ll}
A & B \\
B & D
\end{array}\right]\left\{\begin{array}{l}
\varepsilon \\
\kappa
\end{array}\right\}
$$

where $F$ and $M$ are the resultant force and moment vectors, respectively; $\varepsilon$ and $\kappa$ are the mid-plane strain and curvature vectors, respectively. The matrices of extensional, coupling and bending stiffnesses can be expressed as

$$
([A],[B],[D])=\sum_{k=1}^{N} \int_{h_{k-1}}^{h_{k}}\left(1, z, z^{2}\right)[Q]^{k},
$$

where $h_{\mathrm{k}}$ and $h_{\mathrm{k}-1}$ are the vertical positions of upper and lower surfaces of the $k$ th ply (Fig. 1b). $[Q]^{k}$ are the transformed reduced stiffnesses and $N$ is the ply number.

Considering a symmetric and balanced laminate, the coupling stiffness $[B]$ can be neglected, and the extensional stiffness $[A]$ and flexural stiffness $[D]$ can be expressed as

$$
\left[\begin{array}{l}
A_{11} \\
A_{22} \\
A_{12} \\
A_{66} \\
A_{16} \\
A_{26}
\end{array}\right]=h\left[\begin{array}{ccc}
U_{1} & V_{1 A}^{*} & V_{2 A}^{*} \\
U_{1} & -V_{1 A}^{*} & V_{2 A}^{*} \\
U_{4} & 0 & -V_{2 A}^{*} \\
U_{5} & 0 & -V_{2 A}^{*} \\
0 & 0.5 V_{3 A}^{*} & V_{4 A}^{*} \\
0 & 0.5 V_{3 A}^{*} & -V_{4 A}^{*}
\end{array}\right]\left[\begin{array}{l}
1 \\
U_{2} \\
U_{3}
\end{array}\right],\left[\begin{array}{l}
D_{11} \\
D_{22} \\
D_{12} \\
D_{66} \\
D_{16} \\
D_{26}
\end{array}\right]=\frac{h^{3}}{12}\left[\begin{array}{ccc}
U_{1} & V_{1 D}^{*} & V_{2 D}^{*} \\
U_{1} & -V_{1 D}^{*} & V_{2 D}^{*} \\
U_{4} & 0 & -V_{2 D}^{*} \\
U_{5} & 0 & -V_{2 D}^{*} \\
0 & 0.5 V_{3 D}^{*} & V_{4 D}^{*} \\
0 & 0.5 V_{3 D}^{*} & -V_{4 D}^{*}
\end{array}\right]\left[\begin{array}{l}
1 \\
U_{2} \\
U_{3}
\end{array}\right],
$$

where $U_{\mathrm{i}}(\mathrm{i}=1, \ldots, 5)$ are the material constants, the lamination parameters are

$$
\begin{aligned}
& V_{[1,2,3,4] A}^{k}=\frac{1}{h} \int_{-h / 2}^{h / 2}[\cos 2 \theta, \cos 4 \theta, \sin 2 \theta, \sin 4 \theta] d z=\frac{2}{N} \sum_{k=1}^{N / 2}\left[\cos 2 \theta_{k}, \cos 4 \theta_{k}, \sin 2 \theta_{k}, \sin 4 \theta_{k}\right], \\
& V_{[1,2,3,4] D}^{*}=\frac{12}{h^{3}} \int_{h / 2}^{h / 2} z^{2}[\cos 2 \theta, \cos 4 \theta, \sin 2 \theta, \sin 4 \theta] d z=\frac{8}{N^{3}} \sum_{k=1}^{N / 2}\left[\cos 2 \theta_{k}, \cos 4 \theta_{k}, \sin 2 \theta_{k}, \sin 4 \theta_{k}\right]\left(k^{3}-(k-1)^{3}\right),
\end{aligned}
$$

where $\theta_{\mathrm{k}}$ is the $k$ th ply orientation, $k$ denotes the laminate position from the middle plane to the outermost ply (Fig. 1b). Substituting Eq. (A.4) into Eq. (A.3): 


$$
\begin{aligned}
& A_{11}=h\left[U_{1}+\frac{2 U_{2}}{N} \sum_{k=1}^{N / 2}\left(\cos 2 \theta_{k}\right)+\frac{2 U_{3}}{N} \sum_{k=1}^{N / 2}\left(\cos 4 \theta_{k}\right)\right], \\
& A_{22}=h\left[U_{1}-\frac{2 U_{2}}{N} \sum_{k=1}^{N / 2}\left(\cos 2 \theta_{k}\right)+\frac{2 U_{3}}{N} \sum_{k=1}^{N / 2}\left(\cos 4 \theta_{k}\right)\right], \\
& A_{12}=h\left[U_{4}-\frac{2 U_{3}}{N} \sum_{k=1}^{N / 2}\left(\cos 4 \theta_{k}\right)\right], \\
& A_{16}=h\left[\frac{U_{2}}{N} \sum_{k=1}^{N / 2}\left(\sin 2 \theta_{k}\right)+\frac{2 U_{3}}{N} \sum_{k=1}^{N / 2}\left(\sin 4 \theta_{k}\right)\right], \\
& A_{26}=h\left[\frac{U_{2}}{N / 2} \sum_{k=1}^{N / 2}\left(\sin 2 \theta_{k}\right)-\frac{2 U_{3}}{N} \sum_{k=1}^{N / 2}\left(\sin 4 \theta_{k}\right)\right], \\
& A_{66}=h\left[U_{5}-\frac{2 U_{3}}{N^{3}} \sum_{k=1}^{N / 2}\left(\cos 4 \theta_{k}\right)\right], \\
& D_{11}=\sum_{k=1}^{N / 2}\left(D_{11}\right)_{k}= \sum_{k=1}^{N / 2}\left\{\frac{h^{3} U_{1}}{6 N}+\frac{2 h^{3}}{3 N^{3}}\left(k^{3}-(k-1)^{3}\right)\left[U_{2}\left(\cos 2 \theta_{k}\right)+U_{3}\left(\cos 4 \theta_{k}\right)\right]\right\}, \\
& D_{22}=\sum_{k=1}^{N / 2}\left(D_{22}\right)_{k}=\sum_{k=1}^{N / 2}\left\{\frac{h^{3} U_{1}}{6 N}+\frac{2 h^{3}}{3 N^{3}}\left(k^{3}-(k-1)^{3}\right)\left[-U_{2}\left(\cos 2 \theta_{k}\right)+U_{3}\left(\cos 4 \theta_{k}\right)\right]\right\}, \\
& D_{12}=\sum_{k=1}^{N / 2}\left(D_{12}\right)_{k}=\sum_{k=1}^{N / 2}\left\{\frac{h^{3} U_{4}}{6 N}+\frac{2 h^{3}}{3 N^{3}}\left(k^{3}-(k-1)^{3}\right)\left[-U_{3}\left(\cos 4 \theta_{k}\right)\right]\right\}, \\
& D_{16}=\sum_{k=1}^{N / 2}\left(D_{16}\right)_{k}=\frac{h^{3}}{3 N^{3}} \sum_{k=1}^{N / 2}\left(k^{3}-(k-1)^{3}\right)\left[U_{2} \sin 2 \theta_{k}+2 U_{3} \sin 4 \theta_{k}\right], \\
& D_{26}=\sum_{k=1}^{N / 2}\left(D_{26}\right)_{k}=\frac{h^{3}}{3 N^{3}} \sum_{k=1}^{N / 2}\left(k^{3}-(k-1)^{3}\right)\left[U_{2} \sin 2 \theta_{k}-2 U_{3} \sin 4 \theta_{k}\right], \\
& D_{k=1}^{N / 2}\left(D_{66}\right)_{k}=\sum_{k=1}^{N / 2}\left\{\frac{h^{3} U_{5}}{6 N}+\frac{2 h^{3}}{3 N^{3}}\left(k^{3}-(k-1)^{3}\right)\left[-U_{3}\left(\cos 4 \theta_{k}\right)\right]\right\},
\end{aligned}
$$

Note that in Eq. (A.5), $A_{i j}(i, j=1,2,6)$ are interrelated to the number of the ply orientations, but independent of the stacking sequence. In Eq. (A.6), $D_{\mathrm{ij}}(i, j=1,2,6)$ are expressed as the sum of those parameters at each position $\left(D_{\mathrm{ij}}\right)_{\mathrm{k}}$, which are proportional to the product of the stacking position function $\left(k^{3}-(k-1)^{3}\right)$ and the trigonometric functions of ply orientation $\theta_{\mathrm{k}}$.

\section{Appendix B Example of SPT method}

The detailed searching process of load case 2 with 4 orientations $\left(0^{\circ}, 45^{\circ},-45^{\circ}\right.$, $90^{\circ}$ ) is shown below, where the orientations in red are the positions to satisfying the 
continuity constraints, the orientations in blue are the replaced positions and the orientations in green are the positions that will be replaced.

Step 1: Initialize the sequential permutation table, then choose orientation $\alpha_{\max }=45^{\circ}$ with maximal $\lambda_{c b}=13640.46$, set the initial "Seq" as $\left[( \pm 45)_{12}\right]_{\mathrm{s}}$;

Table B.1 Sequential permutation table of load case 2 with 4 orientations $\left(0^{\circ}, 45^{\circ}\right.$, $\left.-45^{\circ}, 90^{\circ}\right)$

\begin{tabular}{ccccccc}
\hline Steps & Sequence & $\lambda_{c b}$ & $\lambda_{c f}$ & Sequence & $\lambda_{c b}$ & $\lambda_{c f}$ \\
\hline \multirow{3}{*}{ Step 1 } & {$\left[( \pm 45)_{12}\right]_{\mathrm{s}}$} & $\mathbf{1 3 6 4 0 . 4 6}$ & 5952.00 & {$\left[0_{24}\right]_{\mathrm{s}}$} & 5906.23 & $\mathbf{2 5 6 0 0 . 0 0}$ \\
& {$\left[90_{24}\right]_{\mathrm{s}}$} & 12320.80 & 8837.31 & {$\left[90_{24}\right]_{\mathrm{s}}$} & 12320.80 & 8837.31 \\
& {$\left[0_{24}\right]_{\mathrm{s}}$} & 5906.23 & 25600.00 & {$\left[( \pm 45)_{12}\right]_{\mathrm{s}}$} & 13640.46 & 5952.00 \\
\hline
\end{tabular}

Step 2: According to Table B.1, choose the orientation $\beta_{\max }=0^{\circ}$ with the maximal $\lambda_{c f}$.

Use $\beta_{\max }$ to replace $\alpha_{\max }$ from the mid-plane to the outermost ply, set the current position as $k$ and the current sequence as "Seq". The replacement process is shown in

Table B.2.

Step 3: Compare $\lambda_{c b}$ with $\lambda_{c f}$ after each replacement operation in Step 2.

Table B.2 Replacement process from Step 2 to Step 4

\begin{tabular}{|c|c|c|c|c|c|}
\hline Steps & Sequence & $\lambda_{c b}$ & $\lambda_{c f}$ & $\lambda_{c}$ & Compare \\
\hline \multirow{6}{*}{$\begin{array}{c}\text { Step } 2 \text { to } \\
\text { Step } 4\end{array}$} & {$\left[( \pm 45)_{9} / 45 /-45 / 45 /-45 / 45 /-45\right]_{\mathrm{s}}$} & 13640.46 & 5952.00 & 5952.00 & $\lambda_{c b}>\lambda_{c f}$ \\
\hline & {$\left[( \pm 45)_{9} / 45 /-45 / 45 /-45 / 0 / 0\right]_{\mathrm{s}}$} & 13635.99 & 6942.16 & 6942.16 & $\lambda_{c b}>\lambda_{c f}$ \\
\hline & {$\left[( \pm 45)_{8} / 45 /-45 / 45 / 0 / 0 /-45 / 0 / 0\right]_{\mathrm{s}}$} & 13581.19 & 8925.79 & 8925.79 & $\lambda_{c b}>\lambda_{c f}$ \\
\hline & {$\left[( \pm 45)_{7} / 45 /-45 / 45 / 0 / 0 / 0 / 0 /-45 / 0 / 0\right]_{\mathrm{s}}$} & 13459.19 & 10886.20 & 10886.20 & $\lambda_{c b}>\lambda_{c f}$ \\
\hline & {$\left[( \pm 45)_{6} / 45 /-45 / 0 / 0 / 45 / 0 / 0 / 0 / 0 /-45 / 0 / 0\right]_{\mathrm{s}}$} & 13186.17 & 12818.44 & 12818.44 & $\lambda_{c b}>\lambda_{c f}$ \\
\hline & {$\left[( \pm 45)_{6} / 0 / 0 / 0 / 0 / 45 / 0 / 0 / 0 / 0 /-45 / 0 / 0\right]_{\mathrm{s}}$} & 12778.86 & 14716.06 & 12778.86 & $\lambda_{c f}>\lambda_{c b}$ \\
\hline
\end{tabular}

Step 4: In Table B.2: the last sequence that satisfies $\lambda_{c b}>\lambda_{c f}$ is set as Seq1= $\left[( \pm 45)_{6} / 45 /-45 / 0 / 0 / 45 / 0 / 0 / 0 / 0 /-45 / 0 / 0\right]_{\mathrm{s}}$, and $\lambda_{c b 1}=13186.17, \lambda_{c f 1}=12818.44, \lambda_{c 1}=$ 12818.44, $\left(\lambda_{c 1}\right)_{\text {old }}=12818.44$, turn to Step 5; The sequence that satisfies $\lambda_{c f}>\lambda_{c b}$ is set as Seq2 $=\left[( \pm 45)_{6} / 0 / 0 / 0 / 0 / 45 / 0 / 0 / 0 / 0 /-45 / 0 / 0\right]_{s}, \lambda_{c b 2}=12778.86, \lambda_{c f 2}=14716.06, \lambda_{c 2}=$ 
12778.86 and $\left(\lambda_{c 2}\right)_{\text {old }}=12778.86$, turn to Step 7.

Step 5: In "Seq1", $\lambda_{c f 1}<\lambda_{c b 1}$, thus $\lambda_{c f 1}$ can be improved by selecting the orientation $\beta_{1}$ in SPT. To improve $\lambda_{c f 1}$, select the orientation satisfies: $\lambda_{c f 1}\left(0^{\circ}\right)>\lambda_{c f 1}\left(90^{\circ}\right)>\lambda_{c f 1}$

$\left( \pm 45^{\circ}\right)$. So $\beta_{1}=90^{\circ}$, using two $90^{\circ}$ plies to replace a pair of $\pm 45^{\circ}$. Evaluate the values of $\lambda_{c b 1}=13116.67, \lambda_{c f 1}=12678.78, \lambda_{c 1}=12678.78$ and set $\left(\lambda_{c 1}\right)_{\text {new }}=12678.78$.

Step 6: Compare $\left(\lambda_{c 1}\right)_{\text {new }}$ with $\left(\lambda_{c 1}\right)_{\text {old }}$ : because $\left(\lambda_{c 1}\right)_{\text {new }}<\left(\lambda_{c 1}\right)_{\text {old }}$, an optimal sequence is obtained Opt_seq $1=\left[( \pm 45)_{6} / 45 /-45 / 0 / 0 / 45 / 0 / 0 / 0 / 0 /-45 / 0 / 0\right]_{s}$. Step 5 to Step 6 is shown below;

Table B.3 Replacement process from Step 5 to Step 6

\begin{tabular}{llcccl}
\hline Steps & Sequence & $\lambda_{c b}$ & $\lambda_{c f}$ & $\lambda_{c}$ & \multicolumn{1}{c}{ Compare } \\
\hline \multirow{2}{*}{$\begin{array}{l}\text { Step 5 } \\
\text { to }\end{array}$} & {$\left[( \pm 45)_{6} / 45 /-45 / 0 / 0 / 45 / 0 / 0 / 0 / 0 /-45 / 0 / 0\right]_{\mathrm{s}}$} & 13186.17 & 12818.44 & 12818.44 & $\left(\lambda_{c 1}\right)_{\text {old }}=12818.44$ \\
Step 6 & Opt_seq1= & 13116.67 & 12678.78 & 12678.78 & $\left(\lambda_{c 1}\right)_{\text {new }}=12678.78$ \\
& {$\left[( \pm 45)_{6} / 45 /-45 / 0 / 0 / 45 / 0 / 0 / 0 / 0 /-45 / 0 / 0\right]_{\mathrm{s}}$} & 13186.17 & 12818.44 & 12818.44 & $\left(\lambda_{c 1}\right)_{\text {new }}<\left(\lambda_{c 1}\right)_{\text {old }}$ and \\
& & & & & \\
\end{tabular}

Step 7: In "Seq2", $\lambda_{c f 2}>\lambda_{c b 2}$, thus $\lambda_{c b 2}$ can be further improved by selecting the orientations $\alpha_{1}$ in the sequential permutation table that satisfies $\lambda_{c b 2}\left(45^{\circ}\right)>$ $\lambda_{c b 2}\left(90^{\circ}\right)>\lambda_{c b 2}\left(0^{\circ}\right)$. Using the ply with orientation $90^{\circ}$ to replace the one with $0^{\circ}$ without identifying the sequence. The number of the ply orientations $\left(0^{\circ}, 45^{\circ},-45^{\circ}, 90^{\circ}\right)$ changes from $(10,7,7,0)$ to $(9,7,7,1)$. Calculate the new $\lambda_{c f 2}$ according to the number of the ply orientations: $\lambda_{c f 2}=13678.03$. Record the new $\lambda_{c f 2}$ as $\left(\lambda_{c f 2}\right)_{\text {new. }}$.

Step 8: Compare the value of $\left(\lambda_{c f 2}\right)_{\text {new }}=13678.03$ with $\left(\lambda_{c 2}\right)_{\text {old }}=12778.86$, because $\left(\lambda_{c f 2}\right)_{\text {new }}>\left(\lambda_{c 2}\right)_{\text {old }}$, save the number of the ply orientations $(9,7,7,1)$, which will be used for sequence optimization latter.

Step 9: Using the Algorithm 1 to optimize the sequence, where the number of the ply orientations was identified. Select the sequence with maximal $\lambda_{c}$, save the sequence as 
Seq2 $=\left[( \pm 45)_{6} / 45 / 0 / 0 / 0 /-45 / 0 / 0 / 0 / 0 / 90 / 0 / 0\right]_{\mathrm{s}}$ with corresponding $\lambda_{c b 2}=12999.16, \lambda_{c f 2}$ $=13678.03$ and $\lambda_{c 2}=12999.16$. Record the new $\lambda_{c 2}$ as $\left(\lambda_{c 2}\right)_{\text {new }}=12999.16$.

Step 10: Compare $\left(\lambda_{c 2}\right)_{\text {new }}=12999.16$ with $\left(\lambda_{c 2}\right)_{\text {old }}=12778.86$. If $\left(\lambda_{c 2}\right)_{\text {new }}<\left(\lambda_{c 2}\right)_{\text {old }}$, the replacement operation failed, and $\left(\lambda_{c 2}\right)_{\text {old }}$ is the optimal solution. Save this optimal sequence as "Opt_seq2" and its corresponding factors $\lambda_{c b 2}, \lambda_{c f 2}$ and $\lambda_{c 2}$, turn to Step 11; otherwise, the replace operation succeeded. Compare $\lambda_{c b 2}=12999.16$ with $\lambda_{c f 2}$ 13678.03. If $\lambda_{c b 2}<\lambda_{c f 2}, k=k-1$, turn to Step 7; Otherwise, $\lambda_{c b 2}>\lambda_{c f 2}, k=k-1$, turn to

\section{Step 5.}

Thus, turn to Step 7: select an orientation that satisfies: $\lambda_{c b}\left(45^{\circ}\right)>\lambda_{c b}\left(90^{\circ}\right)>$ $\lambda_{c b}\left(0^{\circ}\right)$, so use one $90^{\circ} \mathrm{ply}$ to replace the $0^{\circ} \mathrm{ply}$. The number of the ply orientations $\left(0^{\circ}, 45^{\circ},-45^{\circ}, 90^{\circ}\right)$ changes from $(9,7,7,1)$ to $(8,7,7,2)$. For the number of ply orientations $(8,7,7,2),\left(\lambda_{c f 2}\right)_{\text {new }}=12678.78,\left(\lambda_{c 2}\right)_{\text {old }}=12999.16 ;\left(\lambda_{c f 2}\right)_{\text {new }}<\left(\lambda_{c 2}\right)_{\text {old }}$, there will be no better solution, so Opt_seq2 $=\left[(45)_{6} / 45 / 0 / 0 / 0 /-45 / 0 / 0 / 0 / 0 / 90 / 0 / 0\right]_{s}$.

Step 11: Two optimal solutions "Opt_seq1" and "Opt_seq2" are obtained. The sequence-design strategy is applied to these two sequences to overcome the continuity constraints. Compare the values of $\lambda_{c},\left(\lambda_{c}\right)_{\text {Opt_seq1 }}=12818.44$ with $\left(\lambda_{c}\right)_{\text {Opt_seq } 2}=$ 12999.16. If $\left(\lambda_{c}\right)_{\text {Opt_seq1 }}>\left(\lambda_{c}\right)_{\text {Opt_seq2 }}$, the optimal sequence is "Opt_seq1"; otherwise, $\left(\lambda_{c}\right)_{\text {Opt_seq1 }}<\left(\lambda_{c}\right)_{\text {Opt_seq2 }}$, the optimal sequence is "Opt_seq2". In this example, the optimal sequence is Opt_seq2 (see Table B.4).

Table B.4 Replacement process from Step 7 to Step 11

\begin{tabular}{llcccc}
\hline Steps & Sequence & $\lambda_{c b}$ & $\lambda_{c f}$ & $\lambda_{c}$ & Compare \\
\hline Step 7 to & {$\left[( \pm 45)_{6} / 0 / 0 / 0 / 0 / 45 / 0 / 0 / 0 / 0 /-45 / 0 / 0\right]_{\mathrm{s}}$} & 12778.86 & 14716.06 & 12778.86 & $\left(\lambda_{c f 2}\right)_{\text {new }}>\left(\lambda_{c 2}\right)_{\text {old }}$ \\
Step 8 & Ply numbers $(10,7,7,0)$ changes to $(9,7,7,1)$ & & 13678.03 & & \\
Step 9 & {$\left[( \pm 45)_{6} / 45 /-45 / 90 / 0_{9}\right]_{\mathrm{s}}$} & 12999.16 & 13678.03 & 12999.16 & \\
& & 25 & & &
\end{tabular}


$\left[( \pm 45)_{6} / 45 / 0 / 0 / 0 /-45 / 0 / 0 / 0 / 0 / 90 / 0 / 0\right]_{\mathrm{s}}$

Step 10

Step 7 to $\quad\left[( \pm 45)_{6} / 45 / 0 / 0 / 0 /-45 / 0 / 0 / 0 / 0 / 90 / 0 / 0\right]_{s}$

Step 8 Ply numbers $(9,7,7,1)$ changes to $(8,7,7,2)$

Step 10 Opt_seq2=

$\left[( \pm 45)_{6} / 45 / 0 / 0 / 0 /-45 / 0 / 0 / 0 / 0 / 90 / 0 / 0\right]_{\text {s }}$

Step 11 Opt_seq $=$ Opt_seq2

$$
\left(\lambda_{c 2}\right)_{\text {new }}>\left(\lambda_{c 2}\right)_{\text {old }}
$$

$\begin{array}{lll}12999.16 & 13678.03 & 12999.16\end{array}$

12678.78

$\left(\lambda_{c f 2}\right)_{\text {new }}<\left(\lambda_{c 2}\right)_{\text {old }}$

$12999.16 \quad 13678.03 \quad 12999.16 \quad\left(\lambda_{c}\right)_{\text {Opt_seq } 2}=12999.16$

$\begin{array}{llll}12999.16 & 13678.03 & 12999.16 & \left(\lambda_{c}\right)_{\text {opt_seq1 }}<\left(\lambda_{c}\right)_{\text {Opt_seq } 2}\end{array}$

Finally, in this example the number of evaluations of $\lambda_{c b}$ is 8 and the evaluations of $\lambda_{c f}$

is 9 (the first initial point in SPT is included), another two should been added for evaluation of the sequential permutation table, so $\mathrm{E}\left(\lambda_{c b}\right)=10, \mathrm{E}\left(\lambda_{c f}\right)=11$, as shown in case 2 of Table 3.

\section{References}

[1] Vinson JR, Sierakowski RL. The behaviour of structures composed of composite materials. Dordrecht, Netherlands: Kluwer Academic Publishers; 1987.

[2] Watkins R.I., Morris A.J. A multicriteria objective function optimization scheme for laminated composites for use in multilevel structural optimization schemes. Comput Methods Appl Mech Eng 1987; 60(2): 233-51.

[3] Bruyneel M. A general and effective approach for the optimal design of fiber reinforced composite structures. Compos Sci Tech. 2006; 66: 1303-14.

[4] Lindgaard E, Lund E. Nonlinear buckling optimization of composite structures. Comput Methods Appl Mech Eng 2010; 199: 2319-330.

[5] Ghiasi H,Pasini D,Lessard L. Optimum stacking sequence design of composite materials Part I: Constant stiffness design. Compos Struct. 2009; 90: 1-11.

[6] Ghiasi H, Fayazbakhsh K, Pasini D, Lessard L. Optimum stacking sequence 
design of composite materials Part II: Variable stiffness design. Compos Struct. 2010; 93: 1-13.

[7] Le Riche R, Haftka RT. Optimization of laminate stacking sequence for buckling load maximization by genetic algorithm. AIAA 1993; 31(5): 951-56.

[8] Kogiso N, Watson LT, Gurdal Z, Haftka RT. Genetic algorithms with local improvement for composite laminate design. Struct Opt 1994; 7(4): 207-18.

[9] Le Riche R, Haftka RT. Improved genetic algorithm for minimum thickness composite laminate design. Compos Eng.1995; 5: 143-61.

[10]Pai N, Kawa A, Weng M. Optimization of laminate stacking sequence for failure load maximization using Tabu search. Compos B: Eng 2003; 34(4): 405-13.

[11]Rao ARM, Arvind N. A scatter search algorithm for stacking sequence optimisation of laminate composites. Compos Struct 2005; 70: 383-402

[12]Liu B, Haftka RT, Akgun MA, Todoroki A. Permutation genetic algorithm for stacking sequence design of composite laminates. Comput Methods Appl Mech Eng 2000; 186: 357-72.

[13] Ozgur E, O.Sonmez F. Optimum design of composite laminates for maximum buckling load capacity using simulated annealing. Compos Struct. 2005; 71: $45-52$.

[14]Liu B, Haftka RT, Akgun M.A. Two-level composite wing structural optimization using response surfaces. Struct Multidiscip Opt. 2000; 20: 87-96.

[15]Liu B, Haftka RT, Trompette P. Maximization of buckling loads of composite panels using flexural lamination parameters. Struct Multidiscip Opt 2004; 26 : 
28-36.

[16]Aymerich F, Serra M. Optimization of laminate stacking sequence for maximum buckling load using the ant colony optimization (ACO) metaheuristic. Compos A: Appl Sci Manuf. 2008; 39: 262-272.

[17] Setoodeh S, Abdalla M.M, T. IJsselmuiden S, Gurdal Z. Design of variable-stiffness composite panels for maximum buckling load. Compos Struct. 2009; 87: 109-17.

[18] Irisarri F.-X., Abdalla M.M, Gurdal Z. Improved shepard's method for the optimization of composite structures. AIAA, 2011; 49: 2726-36.

[19]Wang W, Guo SJ, Chang N, Zhao F, Yang W. A modified ant colony algorithm for the stacking sequence optimisation of a rectangular laminate. Struct Multidiscip Opt. 2010; 41: 711-720.

[20]An HC, Chen SY, Huang H. Laminate stacking sequence optimization with strength constraints using two-level approximations and adaptive genetic algorithm. Struct Multidiscip Opt. 2014; 1-16.

[21]Jun W, Rigoberto B. An integrated approach to shape and laminate stacking sequence optimization of free-form FRP shells. Comput Methods Appl Mech Eng 2006;195:4106-123.

[22]Irisarri F.-X., Bassir D.H., Carrere N., Maire J.-F. Multiobjective stacking sequence optimization for laminated composite structures. Compos Sci Tech. 2009; 69: 983-90.

[23] Irisarri F.-X., Lasseigne A, Leroy FH, Riche RL. Optimal design of laminated 
composite structures with ply drops using stacking sequence tables. Compos Struct 2014; 17: 559-569.

[24]Jing Z, Fan XL, Sun Q. Stacking sequence optimization of composite laminates for maximum buckling load using permutation search algorithm. Compos Struct. $2015 ; 121: 225-36$.

[25]Jing Z, Fan XL, Sun Q. Global shared-layer blending method for stacking sequence optimization design and blending of composite structures. Compos B: Eng. 2015; 69: 181-90.

[26]Liu DZ, Toropov VV, Querin OM, Barton DC. Bi-level optimization of blended composite wing panels. J Aircraft 2011; 48: 107-18.

[27]Bloomfield M.W, Diaconu CG, Weaver PM. On feasible regions of lamination parameters for lay-up optimization of laminated composites. Proc R Soc A 2009, 465: 1123-43.

[28]Gubarev F, Kunin V, Pospelov A. Lay-up optimization of laminated composites: Mixed approach with exact feasibility bounds on lamination. 26 Apr 2013: arXiv:1304.7226 [cs.CE].

[29]Seresta O. Buckling, flutter, and postbuckling optimization of composite structures. Phd Thesis. Virginia Polytechnic Institute and State University. 2007 
Figure captions

Fig. 1 (a) Loading and geometry of laminate; (b) stacking-sequence definition

Fig. 2 (a) Convex hull of $\lambda_{c b}$; (b) non-convex hull of $\lambda_{c}$; (c) relationship of $\lambda_{c b}, \lambda_{c f}$ and $\lambda_{c}$

Fig. 3 Initialization evaluation of sequential permutation table

Fig. 4 Illustration of rules to deal with continuity constraints for laminate with 38 plies

Fig. 5 Flowchart of ply-orientation selection strategy

Fig. 6 Illustrations of searching process for 7 load cases with 4 and 12 orientations

Fig. 7 Comparison of optimal load factors for 7 load cases with 4 and 12 angles 
Table captions

Table 1 Sequential permutation table

Table 2 Details of load cases considered for evaluation

Table 3 Optimal stacking sequence using SPT with 4 orientations of $\left(0^{\circ}, 45^{\circ},-45^{\circ}, 90^{\circ}\right)$

Table 4 Optimal stacking sequence using SPT with 12 orientations of $\left(0^{\circ}, \pm 15^{\circ}, \pm 30^{\circ}, \pm 45^{\circ}\right.$,

$\left.\pm 60^{\circ}, \pm 75^{\circ}, 90^{\circ}\right)$

Table 5 Comparative performance of SPT and other methods

Table 6 Efficiency of SPT method 
Table 1 Sequential permutation table

\begin{tabular}{ccccccc}
\hline Case & \multicolumn{2}{c}{ Sorting by buckling load factor $\lambda_{c b}$} & \multicolumn{3}{c}{ Sorting by failure load factor $\lambda_{c f}$} \\
\cline { 2 - 7 } number & Stacking sequence & $\lambda_{c b}$ & $\lambda_{c f}$ & Stacking sequence & $\lambda_{c b}$ & $\lambda_{c f}$ \\
\hline 1 & {$\left[\left(\alpha_{\max }\right)_{\mathrm{n}}\right]_{\mathrm{s}}$} & $\lambda_{c b}\left(\alpha_{\max }\right)$ & $\lambda_{c f}\left(\alpha_{\max }\right)$ & {$\left[\left(\beta_{\max }\right)_{\mathrm{n}}\right]_{\mathrm{s}}$} & $\lambda_{c b}\left(\beta_{\max }\right)$ & $\lambda_{c f}\left(\beta_{\max }\right)$ \\
$\ldots$ & $\ldots$ & $\ldots$ & $\ldots$ & $\ldots$ & $\ldots$ & $\ldots$ \\
$\ldots$ & {$\left[(\alpha)_{\mathrm{n}}\right]_{\mathrm{s}}$} & $\lambda_{c b}(\alpha)$ & $\lambda_{c f}(\alpha)$ & {$\left[(\beta)_{\mathrm{n}}\right]_{\mathrm{s}}$} & $\lambda_{c b}(\beta)$ & $\lambda_{c f}(\beta)$ \\
$\ldots$ & $\ldots$ & $\ldots$ & $\ldots$ & $\ldots$ & $\ldots$ & $\ldots$ \\
{$[M / 2]+1$} & {$\left[\left(\alpha_{\min }\right)_{\mathrm{n}}\right]_{\mathrm{s}}$} & $\lambda_{c b}\left(\alpha_{\min }\right)$ & $\lambda_{c f}\left(\alpha_{\min }\right)$ & {$\left[\left(\beta_{\min }\right)_{\mathrm{n}}\right]_{\mathrm{s}}$} & $\lambda_{c b}\left(\beta_{\min }\right)$ & $\lambda_{c f}\left(\beta_{\min }\right)$ \\
\hline
\end{tabular}


Table 2 Details of load cases considered for evaluation

\begin{tabular}{cccccc}
\hline Case & Number of plies $N$ & $a(\mathrm{~mm})$ & $b(\mathrm{~mm})$ & $F_{\mathrm{x}}(N / \mathrm{cm})$ & $F_{\mathrm{y}}(N / \mathrm{cm})$ \\
\hline 1 & 48 & 50.8 & 12.7 & 1.75 & 0.22 \\
2 & 48 & 50.8 & 12.7 & 1.75 & 0.44 \\
3 & 48 & 50.8 & 12.7 & 1.75 & 0.88 \\
4 & 64 & 50.8 & 12.7 & 1.75 & 0.22 \\
5 & 64 & 50.8 & 12.7 & 1.75 & 0.44 \\
6 & 64 & 50.8 & 12.7 & 1.75 & 0.88 \\
7 & 64 & 50.8 & 12.7 & 1.75 & 1.75 \\
\hline
\end{tabular}




\section{ACCEPTED MANUSCRIPT}

Table 3 Optimal stacking sequence using SPT with 4 orientations of $\left(0^{\circ}, 45^{\circ},-45^{\circ}\right.$, $90^{\circ}$ )

\begin{tabular}{clccccc}
\hline Case & Stacking sequence with continuity constraints & $\lambda_{c b}$ & $\lambda_{c f}$ & $\lambda_{c}$ & \multicolumn{2}{c}{ Evaluations } \\
\hline 1 & {$\left[( \pm 45)_{4} /-45 / 0_{2} / 45 / 0_{4} /-45 / 0_{4} / 45 / 0_{2}\right]_{\mathrm{s}}$} & 15389.61 & 15194.05 & 15194.05 & 11 & 11 \\
\hline 2 & {$\left[( \pm 45)_{6} / 45 / 0_{3} /-45 / 0_{4} / 90 / 0_{2}\right]_{\mathrm{s}}$} & 12999.16 & 13678.03 & 12999.16 & 10 & 11 \\
3 & {$\left[( \pm 45)_{9} /-45 / 0_{2} / 45 / 0_{2}\right]_{\mathrm{s}}$} & 10385.59 & 11248.13 & 10385.59 & 5 & 6 \\
4 & {$\left[ \pm 45 / 0_{2} /-45 / 0_{4} / 45 / 0_{4} /-45 / 0_{4} / 45 / 0_{4} /-45 / 0_{4} / 45 / 0_{2}\right]_{\mathrm{s}}$} & 29497.00 & 26984.31 & 26984.31 & 17 & 17 \\
5 & {$\left[( \pm 45)_{3} /-45 / 0_{2} / 45 / 0_{4} /-45 / 0_{4} / 45 / 0_{4} /-45 / 0_{4} / 45 / 0_{2}\right]_{\mathrm{s}}$} & 25322.68 & 25659.16 & 25322.68 & 14 & 15 \\
6 & {$\left[( \pm 45)_{8} / 0_{3} / 45 / 0_{4} /-45 / 0_{4} / 90 / 0_{2}\right]_{\mathrm{s}}$} & 23252.48 & 22943.18 & 22943.18 & 11 & 11 \\
7 & {$\left[90_{4} / 45 / 90_{4} /-45 / 90_{2} /( \pm 45)_{10}\right]_{\mathrm{s}}$} & 15349.71 & 15824.10 & 15349.71 & 18 & 16 \\
\hline
\end{tabular}




\section{ACCEPTED MANUSCRIPT}

Table 4 Optimal stacking sequence using SPT with 12 orientations of $\left(0^{\circ}, \pm 15^{\circ}, \pm 30^{\circ}\right.$, $\left.\pm 45^{\circ}, \pm 60^{\circ}, \pm 75^{\circ}, 90^{\circ}\right)$

\begin{tabular}{cllcrccc}
\hline Case & Stacking sequence with continuity constraints & $\lambda_{c b}$ & $\lambda_{c f}$ & $\lambda_{c}$ & \multicolumn{2}{c}{ Evaluations } \\
\cline { 4 - 7 } & & & $\lambda_{c b}$ & $\lambda_{c f}$ \\
\hline 1 & {$\left[( \pm 45)_{4} /(-30)_{2} / 30_{2} /(-15)_{2} / 0_{2} / 15 / 0_{4} / 15 / 0_{2}\right]_{\mathrm{s}}$} & 15677.75 & 15783.45 & 15677.75 & 34 & 54 \\
2 & {$\left[( \pm 60)_{5} / \pm 45 / \pm 30 / 0_{4} /-15 / 0_{4} / 15\right]_{\mathrm{s}}$} & 13325.56 & 13403.62 & 13325.56 & 30 & 44 \\
3 & {$\left[( \pm 60)_{8} /( \pm 15)_{4}\right]_{\mathrm{s}}$} & 10603.62 & 10888.57 & 10603.62 & 21 & 29 \\
4 & {$\left[ \pm 30 / 0_{2} /-45 / 0_{4} / 45 / 0_{4} /-45 / 0_{4} / 45 / 0_{4} /-45 / 0_{4} / 45 / 0_{2}\right]_{\mathrm{s}}$} & 28834.33 & 27646.04 & 27646.04 & 24 & 24 \\
5 & {$\left[( \pm 60)_{2} /( \pm 45)_{2} / \pm 30 / 0_{4} /-15 / 0_{4} /-15 / 0_{4} / 15 / 0_{4} / 15 / 0_{2}\right]_{\mathrm{s}}$} & 26294.41 & 26396.86 & 26294.41 & 48 & 68 \\
6 & {$\left[( \pm 60)_{5} / \pm 45 / \pm 30 /( \pm 15)_{9}\right]_{\mathrm{s}}$} & 22983.17 & 23147.63 & 22983.17 & 27 & 35 \\
7 & {$\left[90_{4} /-75 / 90_{4} / 75 /( \pm 45)_{11}\right]_{\mathrm{s}}$} & 15471.80 & 15892.31 & 15471.80 & 32 & 33 \\
\hline
\end{tabular}


Table 5 Comparative performance of SPT and other methods

\begin{tabular}{|c|c|c|c|c|c|c|}
\hline \multirow[b]{2}{*}{ Methods } & \multicolumn{2}{|c|}{ Load Case 1} & \multicolumn{2}{|c|}{ Load Case 2} & \multicolumn{2}{|c|}{ Load Case 3} \\
\hline & Load Factors & $\begin{array}{c}\text { Evaluations, } \\
\text { Reliability }\end{array}$ & Load Factors & $\begin{array}{c}\text { Evaluations, } \\
\text { Reliability }\end{array}$ & Load Factors & $\begin{array}{c}\text { Evaluations, } \\
\text { Reliability }\end{array}$ \\
\hline $\begin{array}{l}\text { Le Riche [7] - } \\
\text { GA }\end{array}$ & $\begin{aligned} \lambda_{c b} & =14168.12 \\
\lambda_{c f} & =13518.66 \\
\lambda_{c} & =13518.66\end{aligned}$ & $\begin{array}{c}2163.4 \\
98.8 \%\end{array}$ & - & - & $\begin{array}{c}\lambda_{c b}=9998.19 \\
\lambda_{c f}=10394.81 \\
\lambda_{c}=9998.19\end{array}$ & $\begin{array}{l}2163.4 \\
98.8 \%\end{array}$ \\
\hline $\begin{array}{l}\text { Kogiso [8] - } \\
\text { Improved GA }\end{array}$ & $\begin{aligned} \lambda_{c b} & =14659.58 \\
\lambda_{c f} & =13518.66 \\
\lambda_{c} & =13518.66\end{aligned}$ & $\begin{array}{l}520 \\
100 \%\end{array}$ & $\begin{aligned} \lambda_{c b} & =12743.45 \\
\lambda_{c f} & =12678.78 \\
\lambda_{c} & =12678.78\end{aligned}$ & $\begin{array}{l}720 \\
96 \%\end{array}$ & $\begin{array}{c}\lambda_{c b}=9998.20 \\
\lambda_{c f}=10398.14 \\
\lambda_{c}=9998.20\end{array}$ & $\begin{array}{r}1646 \\
46 \%\end{array}$ \\
\hline $\begin{array}{l}\text { Le Riche [9] - } \\
\text { Improved GA }\end{array}$ & $\begin{aligned} \lambda_{c b} & =14659.58 \\
\lambda_{c f} & =13518.66 \\
\lambda_{c} & =13518.66\end{aligned}$ & $\begin{array}{l}320 \\
100 \%\end{array}$ & $\begin{aligned} \lambda_{c b} & =12743.45 \\
\lambda_{c f} & =12678.78 \\
\lambda_{c} & =12678.78\end{aligned}$ & $\begin{array}{l}990 \\
100 \%\end{array}$ & $\begin{array}{l}\lambda_{c b}=9998.20 \\
\lambda_{c f}=10398.14 \\
\lambda_{c}=9998.20\end{array}$ & $\begin{array}{l}1570 \\
96 \%\end{array}$ \\
\hline Pai [10] -TS & $\begin{array}{l}\lambda_{c b}=14659.59 \\
\lambda_{c f}=13518.66 \\
\lambda_{c}=13518.66\end{array}$ & $\begin{array}{l}424, \\
100 \%\end{array}$ & $\begin{aligned} \lambda_{c b} & =12743.46 \\
\lambda_{c f} & =12678.78 \\
\lambda_{c} & =12678.78\end{aligned}$ & $\begin{array}{l}499, \\
93 \%\end{array}$ & $\begin{array}{c}\lambda_{c b}=9992.69 \\
\lambda_{c f}=10398.14 \\
\lambda_{c}=9992.69\end{array}$ & $\begin{array}{l}695 \\
84 \%\end{array}$ \\
\hline Rao [11] -SS & $\begin{array}{l}\lambda_{c b}=14659.46 \\
\lambda_{c f}=13518.67 \\
\lambda_{c}=13518.66\end{array}$ & $\begin{array}{c}- \\
100 \%\end{array}$ & $\begin{aligned} \lambda_{c b} & =12746.93 \\
\lambda_{c f} & =12689.08 \\
\lambda_{c} & =12689.08\end{aligned}$ & $100 \%$ & $\begin{array}{c}\lambda_{c b}=9998.70 \\
\lambda_{c f}=10403.75 \\
\lambda_{c}=9998.70\end{array}$ & $\begin{array}{c}- \\
100 \%\end{array}$ \\
\hline $\begin{array}{c}\text { Aymerich [16] } \\
\text {-ACO }\end{array}$ & $\begin{array}{l}\lambda_{c b}=14659.58 \\
\lambda_{c f}=13518.66 \\
\lambda_{c}=13518.66\end{array}$ & $\begin{array}{l}303, \\
100 \%\end{array}$ & $\begin{aligned} \lambda_{c b} & =12743.45 \\
\lambda_{c f} & =12678.78 \\
\lambda_{c} & =12678.78\end{aligned}$ & $\begin{array}{l}549 \\
81 \%\end{array}$ & $\begin{array}{c}\lambda_{c b}=9998.20 \\
\lambda_{c f}=10398.14 \\
\lambda_{c}=9998.20\end{array}$ & $\begin{array}{l}625 \\
77 \%\end{array}$ \\
\hline $\begin{array}{l}\text { Wang [19] - } \\
\text { MCLACA }\end{array}$ & $\begin{array}{l}\lambda_{c b}=13818.29 \\
\lambda_{c f}=13518.66 \\
\lambda_{c}=13518.66\end{array}$ & $\begin{array}{l}130.4 \\
100 \%\end{array}$ & $\begin{aligned} \lambda_{c b} & =12743.45 \\
\lambda_{c f} & =12678.78 \\
\lambda_{c} & =12678.78\end{aligned}$ & $\begin{array}{l}515.4 \\
85 \%\end{array}$ & $\begin{array}{c}\lambda_{c b}=9998.20 \\
\lambda_{c f}=10398.14 \\
\lambda_{c}=9998.20\end{array}$ & $\begin{array}{c}544.8 \\
94 \%\end{array}$ \\
\hline $\begin{array}{l}\text { An [20] - } \\
\text { AGATLA }\end{array}$ & $\begin{array}{l}\lambda_{c b}=15359.28 \\
\lambda_{c f}=13518.66 \\
\lambda_{c}=13518.66\end{array}$ & $96 \%$ & $\begin{aligned} \lambda_{c b} & =12940.93 \\
\lambda_{c f} & =13378.41 \\
\lambda_{c} & =12940.93\end{aligned}$ & $\begin{array}{l}31.9 \\
93 \%\end{array}$ & $\begin{aligned} \lambda_{c b} & =10089.30 \\
\lambda_{c f} & =10398.14 \\
\lambda_{c} & =10089.30\end{aligned}$ & $\begin{array}{c}25.64 \\
97 \%\end{array}$ \\
\hline SPT & $\begin{aligned} \lambda_{c b} & =15389.61 \\
\lambda_{c f} & =15194.05 \\
\lambda_{c} & =15194.05\end{aligned}$ & $\begin{array}{c}11 \\
100 \%\end{array}$ & $\begin{aligned} \lambda_{c b} & =12999.16 \\
\lambda_{c f} & =13678.03 \\
\lambda_{c} & =12999.16\end{aligned}$ & $\begin{array}{c}10 \\
100 \%\end{array}$ & $\begin{array}{c}\lambda_{c b}=10385.59 \\
\lambda_{c f}=11248.13 \\
\lambda_{c}=10385.59\end{array}$ & $\begin{array}{c}5, \\
100 \%\end{array}$ \\
\hline
\end{tabular}

The data of references $[7-11,16,19,20]$ are getting from the original articles, respectively. 
Table 6 Efficiency of SPT method

\begin{tabular}{cccc}
\hline Number of ply orientations & Stacking position & Design space & Evaluations using SPT \\
\hline \multirow{2}{*}{4} & 24 (48 with 2 stack together) & $4^{12}=1.6777 \times 10^{7}$ & - \\
& 48 & $4^{24}=2.8147 \times 10^{14}$ & $<15$ \\
& 64 & $4^{32}=1.8447 \times 10^{19}$ & $<20$ \\
\hline \multirow{2}{*}{12} & 48 & $12^{24}=7.9497 \times 10^{25}$ & $<60$ \\
& 64 & $12^{32}=3.4182 \times 10^{34}$ & $<70$ \\
\hline
\end{tabular}



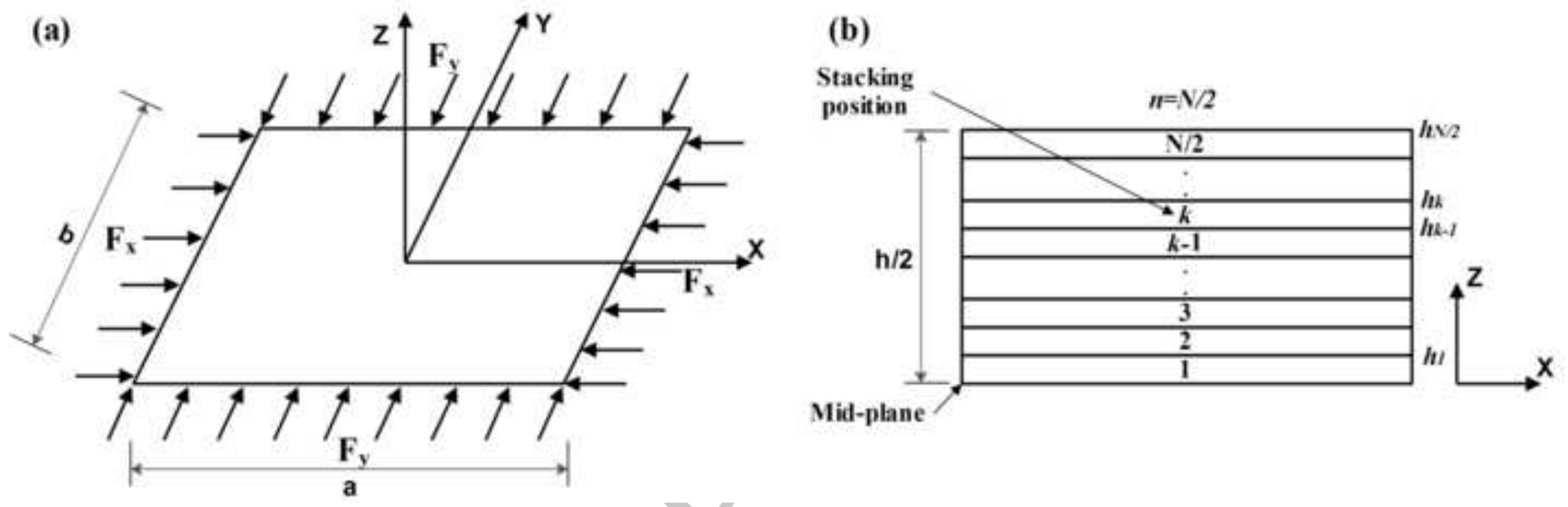

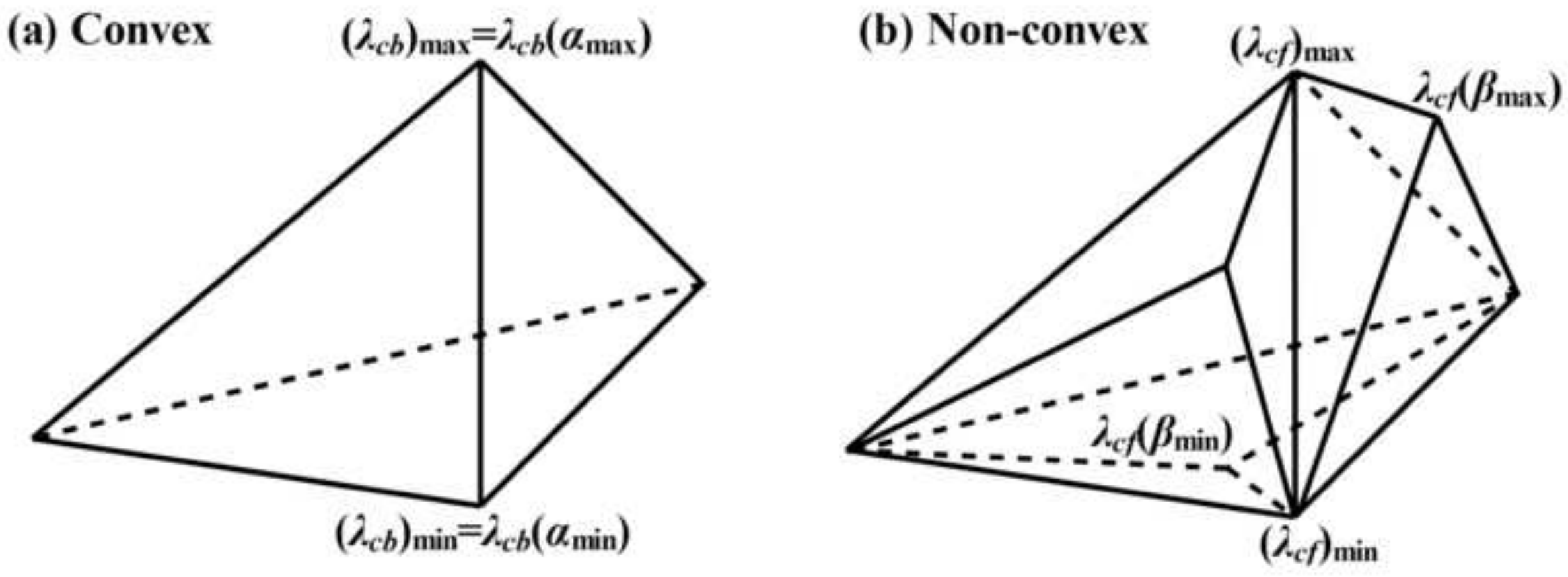

(c) Relationship of $\lambda_{c b}, \lambda_{c f}$ and $\lambda_{c}$

Number of ply orientations

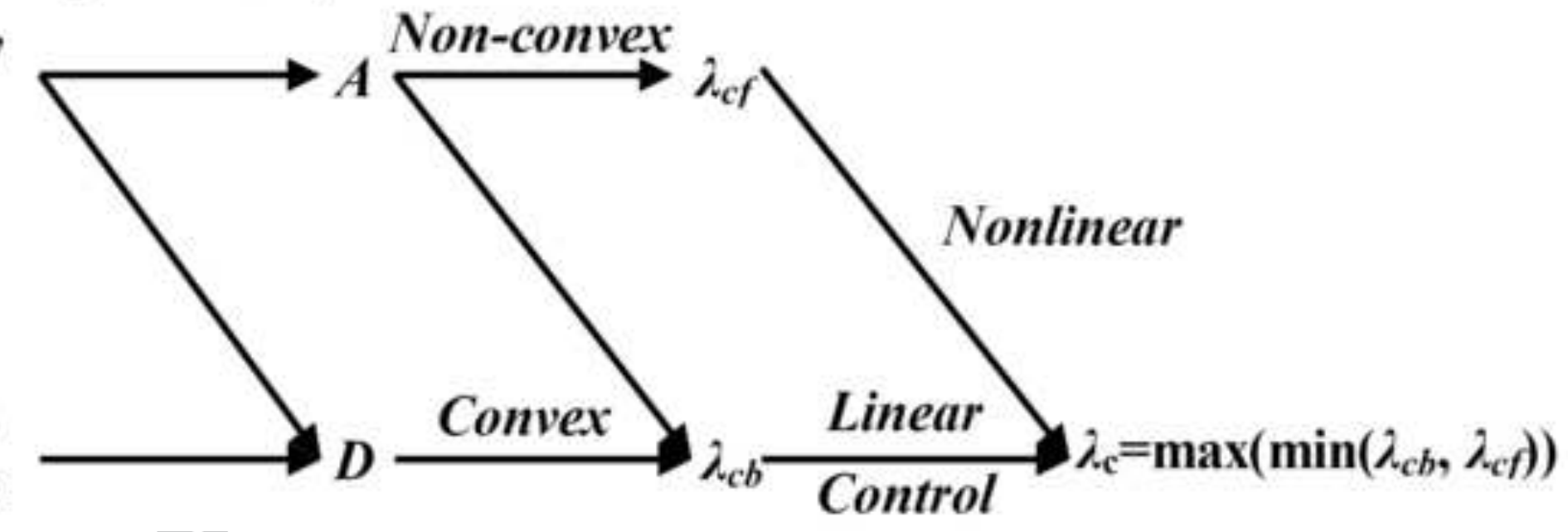

Stacking sequence 
$M$ orientations: $\left\{\theta_{1}, \theta_{2}, \ldots, \theta_{\mathrm{M}}\right\}$, choose one orientation $\theta \in\left\{\vec{\theta}_{1}, \theta_{2}, \ldots, \theta_{\mathrm{M}}\right\}$

Set the sequence with one orientation $\theta$ or pairs of $\pm \theta$

case $1:[\theta / \theta / \theta / \theta \cdots \theta / \theta \cdots \theta / \theta]_{\mathrm{s}} \quad$ if $\theta=0^{\circ}$ or $90^{\circ}$

case 2: $[ \pm \theta / \pm \theta \cdots \pm \theta / \cdots / \pm \theta]_{\mathrm{s}} \quad$ if $\theta \neq 0^{\circ}$ or $90^{\circ}$ 
$\downarrow$ Violate position

$\downarrow$ Most close position with different orientation $\lambda_{c b}\left(0^{\circ}\right)>\lambda_{c b}\left(45^{\circ}\right)=\lambda_{c b}\left(-45^{\circ}\right)>\lambda_{c h}\left(90^{\circ}\right)$

Delete position

Insert position

$\left(N_{0}, N_{45}, N .45, N_{90}\right)_{\text {oripion }}=(12,2,2,3)$

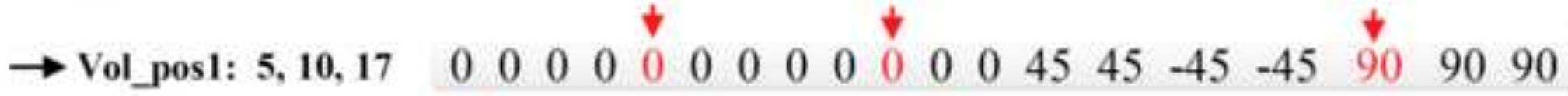

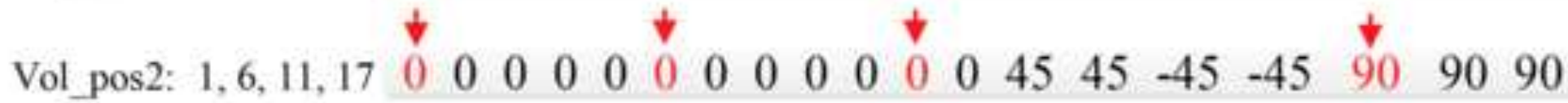

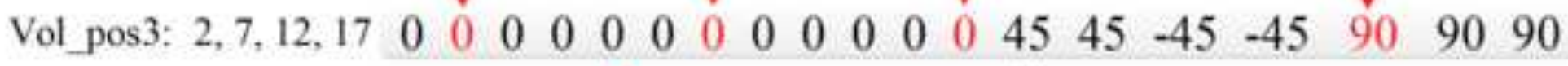
Seq 1: $\begin{array}{lllllllllllllllllll}0 & 0 & 0 & 0 & 0 & 0 & 0 & 0 & 0 & 0 & 0 & 0 & 45 & 45 & -45 & -45 & 90 & 90 & 90\end{array}$

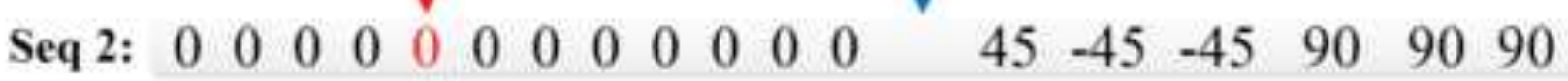
Seq 3: $\begin{array}{lllllllllllllllllll}0 & 0 & 0 & 0 & 45 & 0 & 0 & 0 & 0 & 0 & 0 & 0 & 0 & 45 & -45 & -45 & 90 & 90 & 90\end{array}$

Seq 4: $\begin{array}{lllllllllllllllllll}0 & 0 & 0 & 0 & 45 & 0 & 0 & 0 & 0 & 0 & 0 & 0 & 0 & 45 & -45 & -45 & 90 & 90 & 90\end{array}$ Seq 5: $\begin{array}{llllllllllllllllll}0 & 0 & 0 & 0 & 45 & 0 & 0 & 0 & 0 & 0 & 0 & 0 & 0 & -45 & -45 & 90 & 90 & 90\end{array}$ Seq 6: $\begin{array}{lllllllllllllllllll}0 & 0 & 0 & 0 & 45 & 0 & 0 & 0 & 0 & 45 & 0 & 0 & 0 & 0 & -45 & -45 & 90 & 90 & 90\end{array}$ Seq 7: $\begin{array}{lllllllllllllllllllllllll}0 & 0 & 0 & 0 & 45 & 0 & 0 & 0 & 0 & 45 & 0 & 0 & 0 & 0 & -45 & -45 & 90 & 90 & 90\end{array}$ Seq 8: $\begin{array}{llllllllllllllllll}0 & 0 & 0 & 0 & 45 & 0 & 0 & 0 & 0 & 45 & 0 & 0 & 0 & 0 & -45 & 90 & 90 & 90\end{array}$ Seq 9: $\begin{array}{lllllllllllllllllll}0 & 0 & 0 & 0 & 45 & 0 & 0 & 0 & 0 & 45 & 0 & 0 & 0 & 0 & -45 & 90 & -45 & 90 & 90\end{array}$ 
$+1$

The green orientation is the detected position;

The blue orientation is the replaced position;

The red orientation is the position to overcome the continuity constraints.

\section{StepI:Initialization}

$+k$

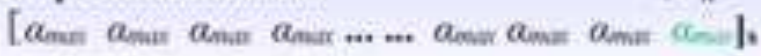
evaluate $\lambda_{e}$, $\lambda_{0}$ and $\lambda_{\text {. }}$

\section{Step 2: Replacement operation $\leftarrow \ell$}

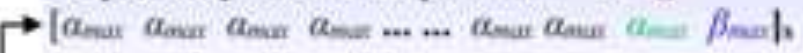

evaluate $\lambda_{d} \lambda_{\text {ef }}$ and $\lambda_{c}$.

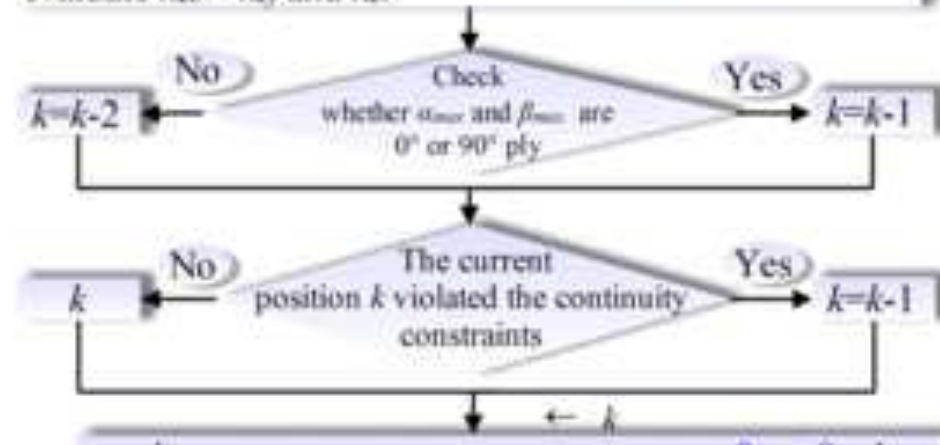

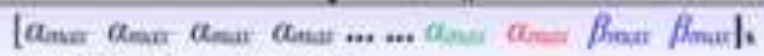
evaluate $\lambda_{e}, \lambda_{0}$ and $\lambda_{0}$.

No)

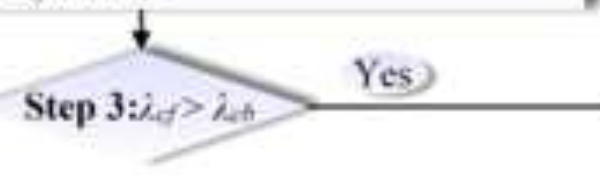

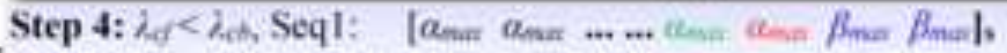

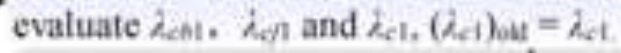

Step 5: Select the orientation $\beta_{1}: \lambda_{n-1}\left(\beta_{\max }\right)>\lambda_{-n}\left(\beta_{1}\right)>\lambda_{\mathrm{c} n}\left(a_{\text {nasa }}\right)$

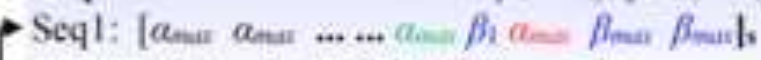

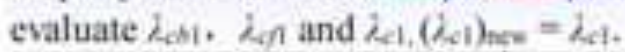
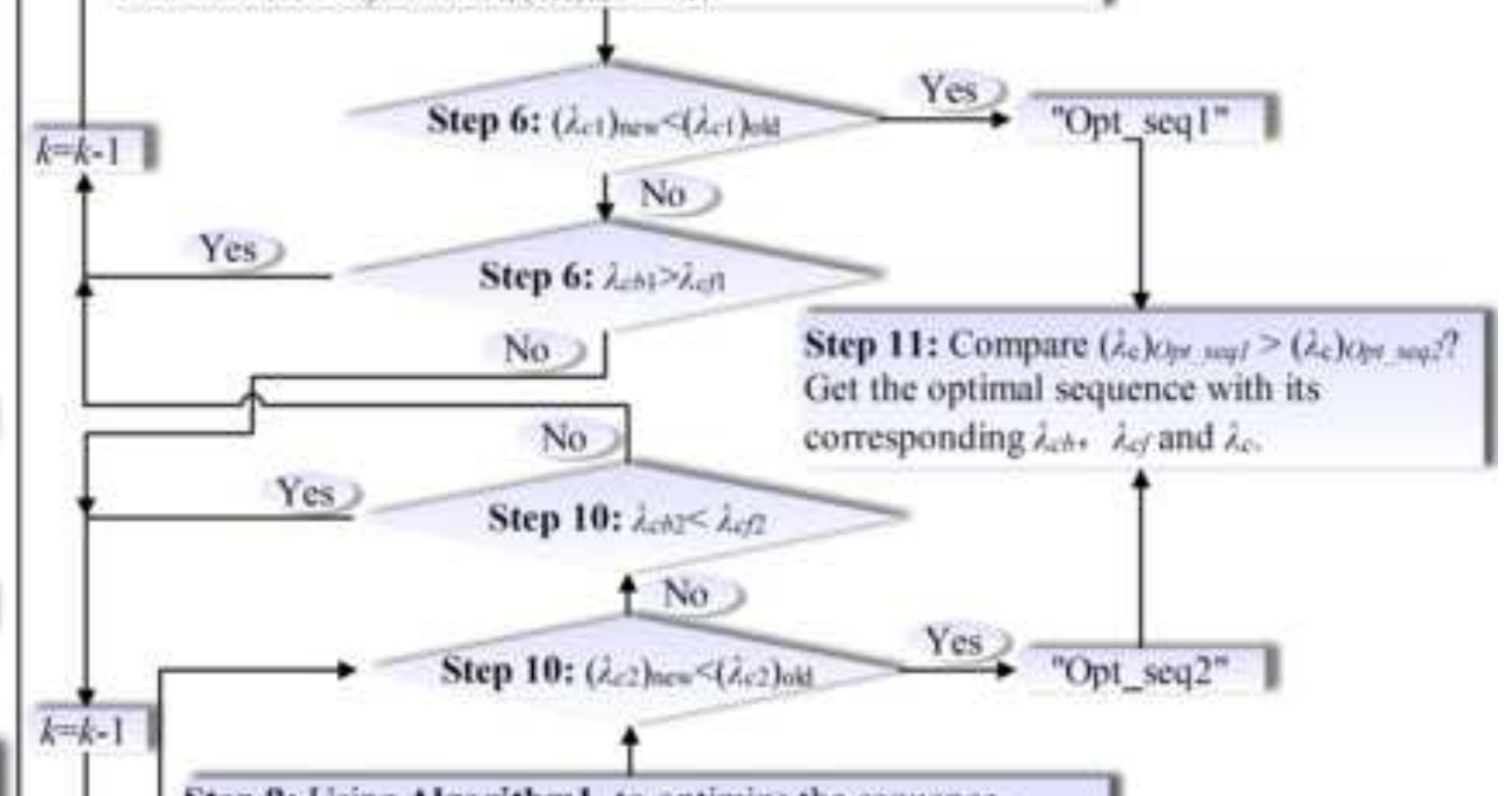

Step 9: Using AlgorithmI to optimize the sequence,

Select the sequence with maximal $i$, save the sequence as

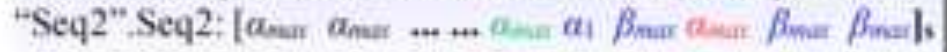

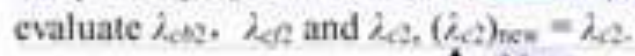

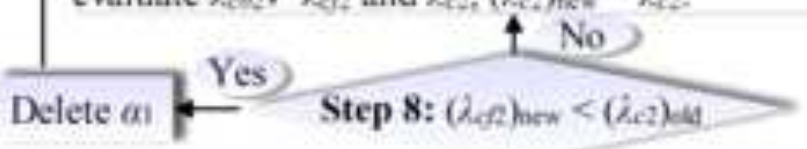

\section{$\uparrow$}

Step 7: Select the orientation $\alpha_{1}: \lambda_{\text {enz }}\left(\alpha_{\operatorname{man}}\right)>\lambda_{\text {ang }}\left(\alpha_{1}\right)>\lambda_{4}+2\left(\beta_{\operatorname{mat}}\right)$.

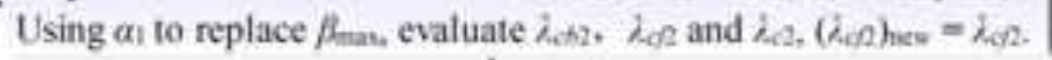
$\uparrow \leftarrow k$

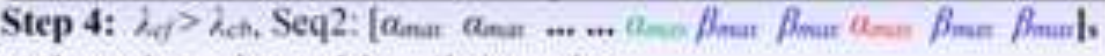

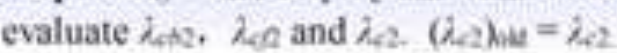




\section{ACCEPTED MANUSCRIPT}
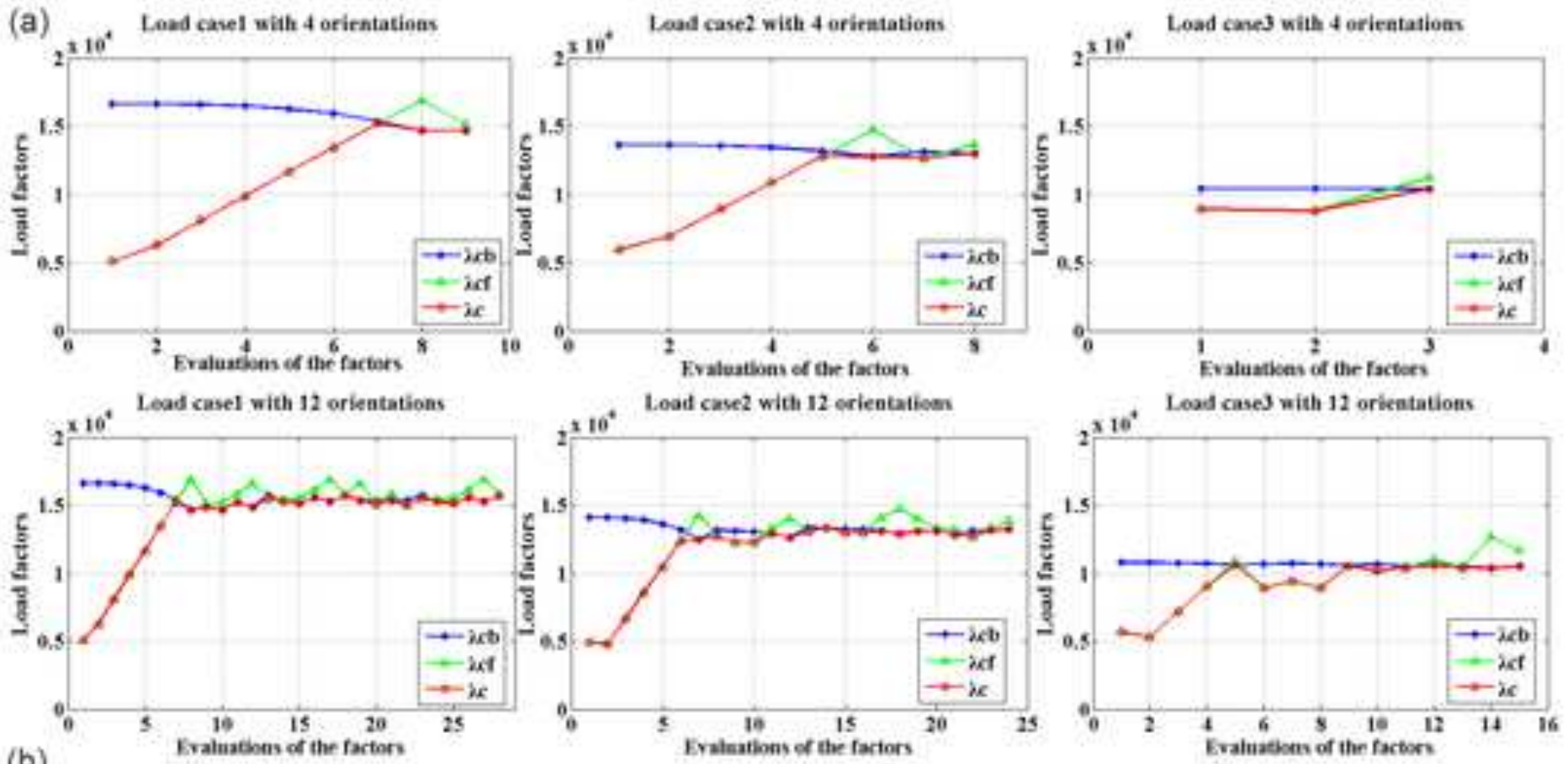

(b)
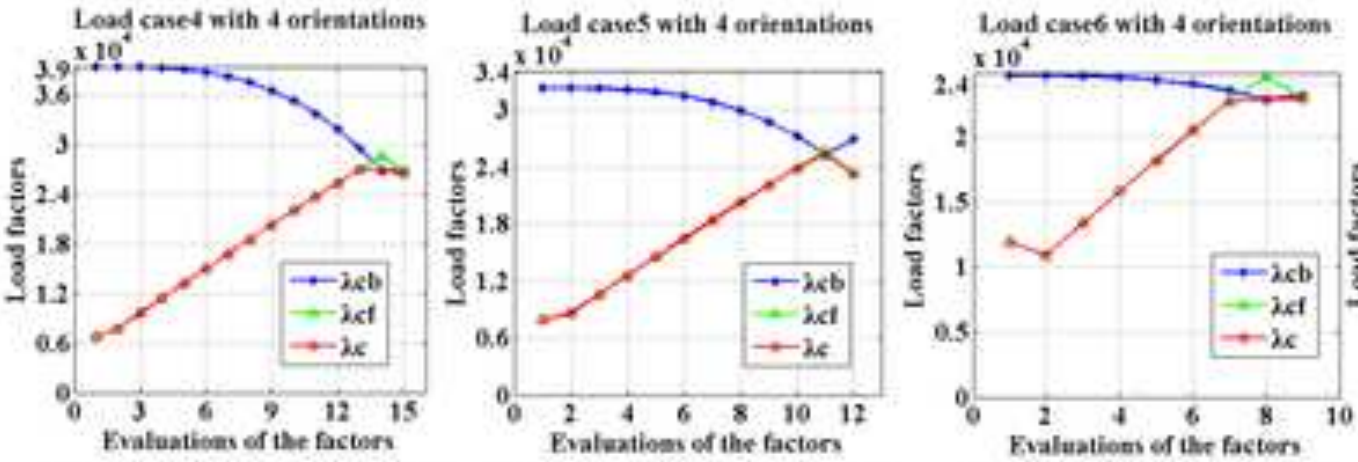

Load case7 with 4 oriestations
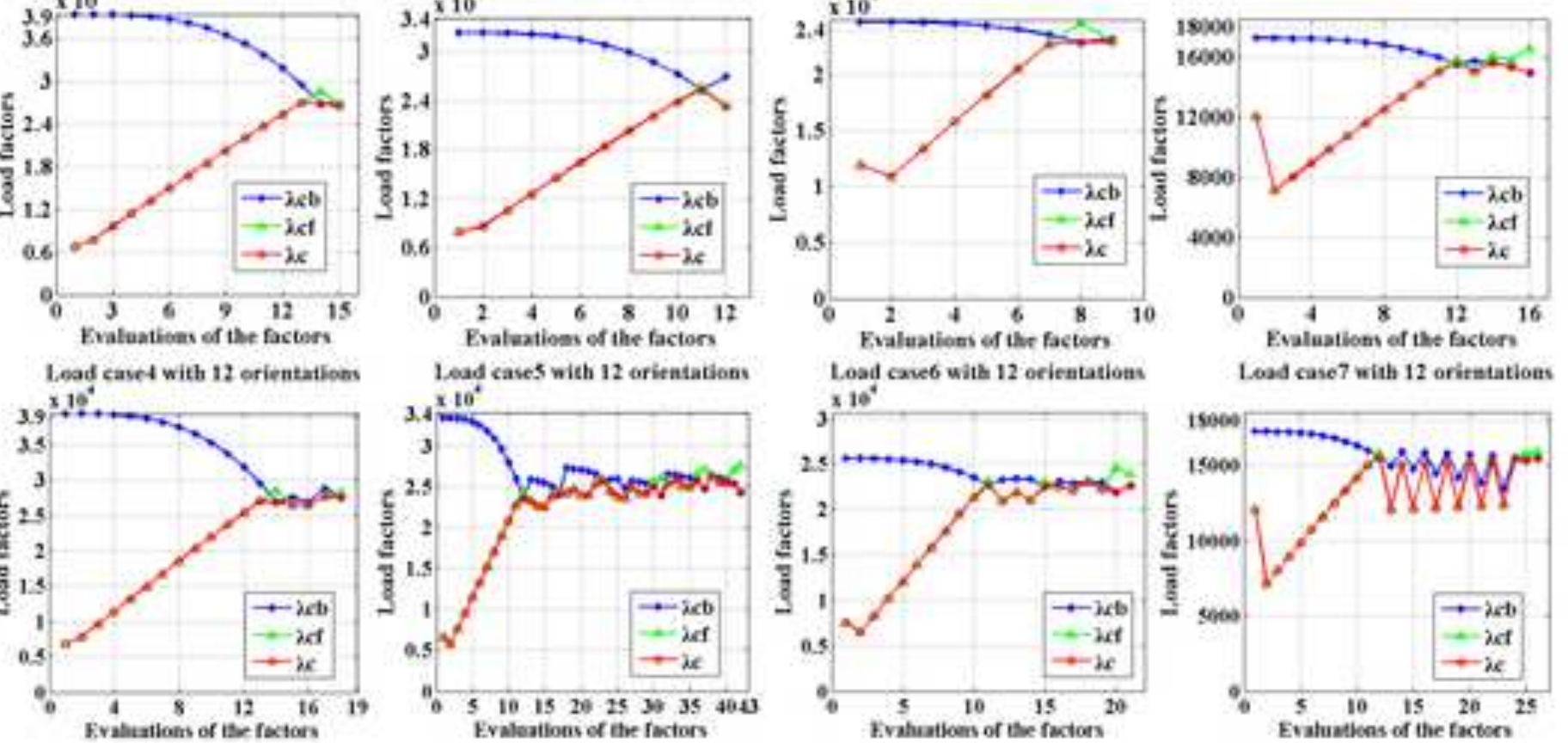

Logd case 7 with 12 oritatatioas

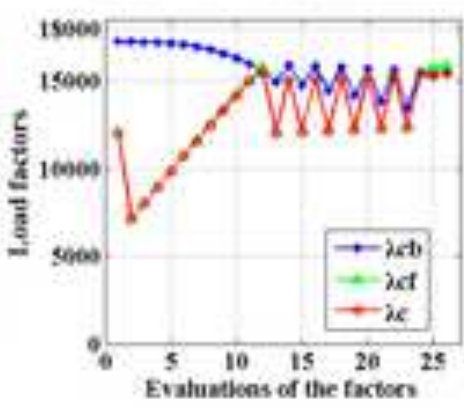




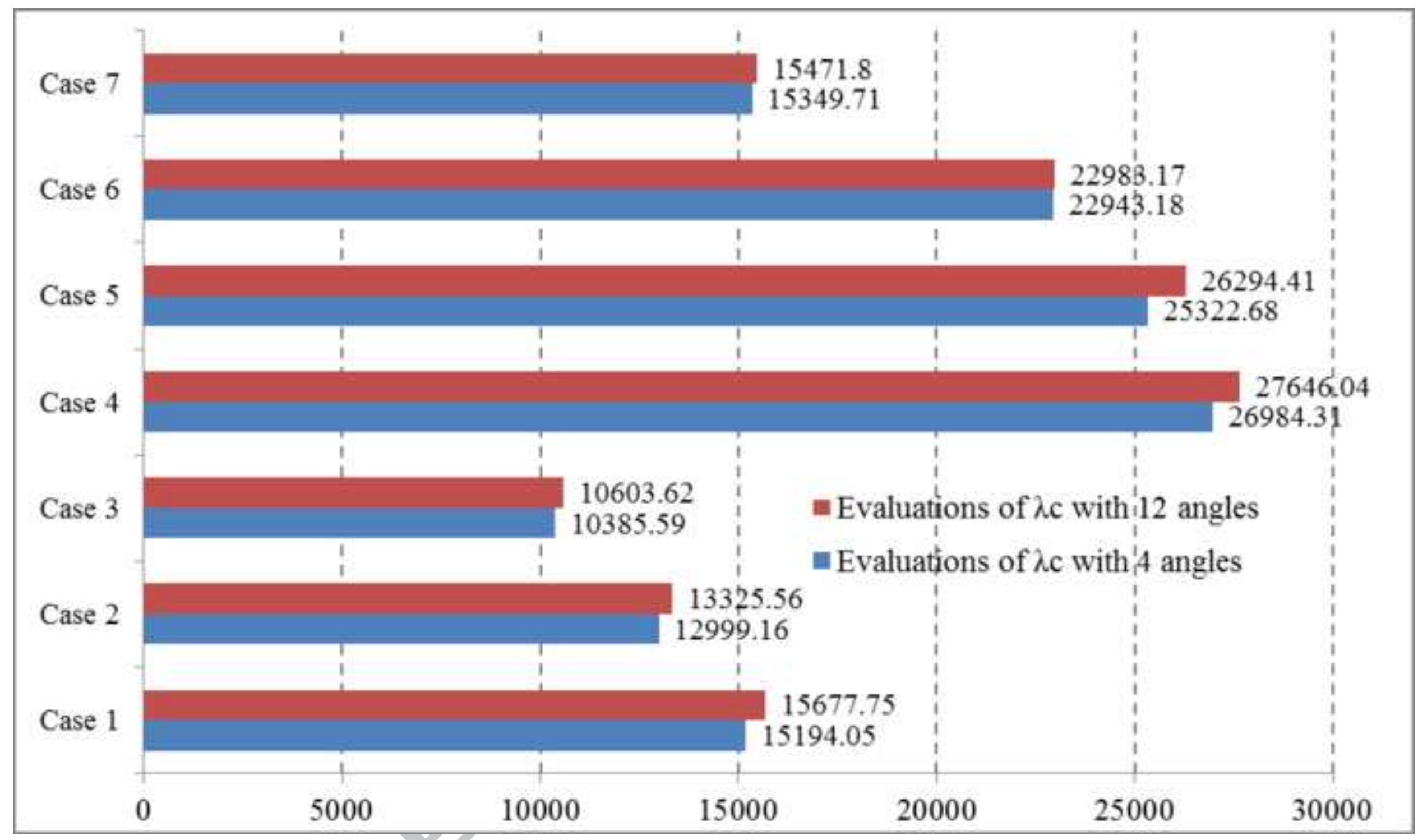

